\title{
The Normalized-Rate Iterative Algorithm: A Practical Dynamic Spectrum Management Method for DSL
}

\author{
Driton Statovci, Tomas Nordström, and Rickard Nilsson \\ Telecommunications Research Center Vienna (ftw.), Donau-City-Straße 1, A-1220 Vienna, Austria
}

Received 17 December 2004; Revised 1 June 2005; Accepted 2 June 2005

\begin{abstract}
We present a practical solution for dynamic spectrum management (DSM) in digital subscriber line systems: the normalized-rate iterative algorithm (NRIA). Supported by a novel optimization problem formulation, the NRIA is the only DSM algorithm that jointly addresses spectrum balancing for frequency division duplexing systems and power allocation for the users sharing a common cable bundle. With a focus on being implementable rather than obtaining the highest possible theoretical performance, the NRIA is designed to efficiently solve the DSM optimization problem with the operators' business models in mind. This is achieved with the help of two types of parameters: the desired network asymmetry and the desired user priorities. The NRIA is a centralized DSM algorithm based on the iterative water-filling algorithm (IWFA) for finding efficient power allocations, but extends the IWFA by finding the achievable bitrates and by optimizing the bandplan. It is compared with three other DSM proposals: the IWFA, the optimal spectrum balancing algorithm (OSBA), and the bidirectional IWFA (bi-IWFA). We show that the NRIA achieves better bitrate performance than the IWFA and the bi-IWFA. It can even achieve performance almost as good as the OSBA, but with dramatically lower requirements on complexity. Additionally, the NRIA can achieve bitrate combinations that cannot be supported by any other DSM algorithm.
\end{abstract}

Copyright (c) 2006 Driton Statovci et al. This is an open access article distributed under the Creative Commons Attribution License, which permits unrestricted use, distribution, and reproduction in any medium, provided the original work is properly cited.

\section{INTRODUCTION}

In the development of currently deployed digital subscriber line (DSL) systems, a single user scenario was assumed with worst case crosstalk models. This passive strategy was motivated by the goal of maximizing the robustness of DSL systems. In practice, however, it often leads to overly conservative performance figures and sometimes even to failures to deliver a specific DSL service. This is often due to a poorly optimized resource allocation among different loops in a cable bundle, combined with unmotivated high noise margins, which result in too pessimistic bitrates. Furthermore, if the system environment changes in a practical scenario, for example, where unmodelled noise sources appear, the initial robustness of a static deployed DSL system easily breaks down.

With an active approach to copper bundle resource management, the cable resources can be more efficiently shared among the users. Combined with more accurate crosstalk figures obtained from accurate online cable measurements, higher and more balanced bitrates can be achieved on most loops. In the literature, this is often referred to as dynamic spectrum management (DSM), although usually only active power control over a predefined static spectrum bandplan between the downstream and upstream is considered.

In this paper, we describe a practically applicable DSM method for DSL called the normalized-rate iterative algorithm (NRIA) [1-3]. The NRIA is a centralized DSM algorithm based on the iterative water-filling algorithm (IWFA) [4] for finding good power allocations, but it extends the IWFA by automatically deriving achievable bitrates and searching for an optimized bandplan.

The NRIA jointly balances the spectrum between the downstream and upstream directions, that is, it finds an effective common bandplan for frequency division duplexing (FDD) DSLs, and performs power allocation for each line in a cable bundle. The NRIA is suboptimal in the sense that the power allocation is based on the IWFA, and the frequency allocation is based on an ad hoc solution.

Compared to other DSM methods, the NRIA has essentially two major advantages: high performance and low complexity. Since the NRIA optimizes the bandplan, better performance can be achieved than with the IWFA, which uses a static (fixed) bandplan. Compared to the optimal spectrum balancing algorithm (OSBA) [5-7], which in theory can deliver the highest bitrates for a given bandplan, the NRIA can 
in practice achieve almost as good performance but with a dramatically lower requirement on complexity. This is crucial especially for the more realistic cases where the number of loops in a bundle is more than a couple. In this case, since the complexity of the OSBA grows exponentially with the number of loops, it effectively fails to deliver any result in a reasonable time. Furthermore, due to the extended capability of searching for an optimized bandplan, the NRIA supports several downstream and upstream bitrate combinations that cannot be supported by any other DSM algorithm.

When using the IWFA the achievable bitrates needs to be specified in advance (before running the algorithm). Since this is difficult the NRIA has an important advantage as it finds the achievable bitrates automatically and needs no prior knowledge about them. Furthermore, tractable operating points for desirable business models can easily be achieved, because the NRIA has a parameter for selecting the desired downstream and upstream asymmetry and parameters for selecting the user priorities.

As with the OSBA, a potential drawback of the NRIA, however, is that it is a centralized algorithm operated by a common DSM agent. Nevertheless, in practice a DSM agent may always be necessary since the users' bitrates that can be supported by a distributed DSM algorithm like the IWFA must be calculated by a central agent.

The paper is organized as follows: Section 2 describes the system model; Section 3 describes some fundamental bitrate relations used in Section 4, which formulates the multiuser optimization problem that we consider; Section 5 describes the NRIA as a suboptimal but practical solution to the problem; Section 6 presents simulation results of the NRIA with comparisons to the IWFA, the OSBA, and the bidirectional IWFA [8]; and Section 7 summarizes the major findings presented in this paper.

\section{SYSTEM MODEL}

Figure 1 shows a typical network scenario that the NRIA is designed to handle. Specifically, it is assumed that both ends of the cable can be distributed; at the line termination (LT) side, the loops can be connected to a central office (CO) as well as a cabinet; and at the network termination (NT) side, the loops are connected to customer premises equipment (CPE), which are usually distributed in space.

The crucial assumption with this network model is that all loops have unique crosstalk couplings between each other. This assumption is valid in practice. Even if some cables are collocated at one end, or even at both ends, they will still have different crosstalk couplings due to other differences such as in the twists, the locations of the loops within a bundle, and the loop termination at the NT and LT sides. For example, it is not always the case that the longest loop in a network has the poorest DSL channel conditions.

To make efficient dynamic spectral balancing possible, high flexibility in selecting the transmission spectra is needed by the DSL systems. Multicarrier modulation combined with digital frequency division duplexing (D-FDD), like the Zipper [9] duplexing method based on discrete multitone

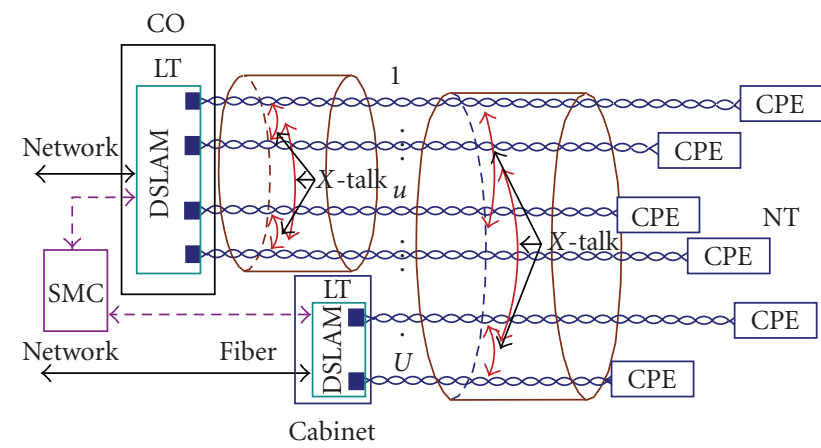

FIGURE 1: A distributed multiuser DSL environment with customer premises equipment (CPE) connected to a central office $(\mathrm{CO})$ as well as to a cabinet. SMC denotes the spectrum management center. DSLAM denotes DSL access multiplexer. LT and NT denote line termination and network termination sides, respectively.

modulation (DMT), offers this flexibility. Since Zipper-DMT is also part of current VDSL standards, we assume that it is used. Furthermore, full network synchronization is also assumed [10] in order to avoid any efficiency loss due to silent guard bands between the downstream and upstream subbands, to avoid flexibility loss in frequency planning [11], and to make crosstalk noise on different subcarriers independent. $^{1}$

The NRIA relies on a spectrum management center (S$\mathrm{MC}$ ), as shown in Figure 1. Firstly, the SMC collects all channel characteristics in the network from individual modems, including the crosstalk channels, during an offline period. Secondly, it runs the NRIA in order to find a common DFDD bandplan and individual power transmit spectra for each modem. Finally, these parameters are returned by the SMC to every modem before they start to operate.

With network synchronization of a Zipper-DMT system, a received symbol after the DFT demodulator on subcarrier $n$ for user $u, Y_{u}^{n}$, can be expressed as

$$
Y_{u}^{n}=H_{u u}^{n} X_{u}^{n}+\sum_{\substack{v=1 \\ v \neq u}}^{U} H_{u v}^{n} X_{v}^{n}+V_{u}^{n}, \quad u \in\{1, \ldots, U\}
$$

where $X_{u}^{n}$ and $X_{v}^{n}$ are the transmitted symbols of user $u$ and user $v$ on subcarrier $n$, respectively. $V_{u}^{n}$ is the background noise of user $u$ on subcarrier $n$. $H_{u v}^{n}$ is the channel transfer function from user $v$ to user $u$, that is, it represents either the direct channel (with $v=u$ ), or far-end crosstalk (FEXT).

Note that with synchronization and D-FDD, that is, synchronous Zipper, the near end crosstalk (NEXT) is completely eliminated through orthogonality, regardless of the selected bandplan (downstream and upstream subcarriers).

\footnotetext{
${ }^{1}$ Without network synchronization, signal energy assigned by one user on one subcarrier will also leak over to neighboring subcarriers for the other users, due to the asynchrony, and thus appear as nonorthogonal near-end crosstalk and far-end crosstalk.
} 
Therefore, the desired symbol $X_{u}^{n}$ is only disturbed by FEXT, which originates from all other users from the corresponding subcarrier $n$, and the background noise.

The number of bits in a DMT symbol for user $u$ in the upstream transmission direction is

$$
R_{u, \mathrm{US}}=\sum_{n \in I_{\mathrm{US}}} R_{u}^{n},
$$

where $R_{u}^{n}$ is the number of bits for user $u$ on subcarrier $n$ and $I_{\mathrm{US}}$ represents the set of upstream subcarrier indices. The number of downstream bits $R_{u, \mathrm{DS}}$, is derived correspondingly. To calculate the number of bits that are transmitted per second the $R_{u \text {,Us }}$ is multiplied with the number of DMT symbols that are transmitted in one second. The NRIA calculates an optimized D-FDD bandplan as one part of the DSM process. That is, it finds $R_{u, \mathrm{DS}}$ and $R_{u, \mathrm{US}}$ by iteratively redirecting subcarriers to the downstream or upstream direction.

Let us denote the squared magnitude of the channel transfer function from user $v$ to $u$ on subcarrier $n$ by

$$
\mathscr{H}_{u v}^{n}=\left|H_{u v}^{n}\right|^{2}
$$

Based on the Shannon capacity formula, the number of bits loaded on subcarrier $n$ by user $u$, for two-dimensional symbols, is

$$
R_{u}^{n}=\log _{2}\left(1+\frac{\mathcal{H}_{u u}^{n} \mathcal{P}_{u}^{n}}{\Gamma \mathcal{N}_{u}^{n}}\right)
$$

where $\mathcal{P}_{u}^{n}$ denotes the power spectral density (PSD) of the signal. The PSD specifies the signal power allocation versus frequency. $\mathcal{N}_{u}^{n}$ denotes the PSD of the noise on subcarrier $n$. $\Gamma$ is the signal-to-noise (SNR) gap, which for a given bit error rate and signal constellation represents the loss compared to the Shannon channel capacity. The PSD of noise is calculated by

$$
\mathcal{N}_{u}^{n}=\sum_{\substack{v=1 \\ v \neq u}}^{U} \mathcal{H}_{u v}^{n} \mathcal{P}_{v}^{n}+\mathcal{P}_{V}^{n}
$$

where $\mathcal{P}_{V}^{n}$ denotes the PSD of the background noise on subcarrier $n$.

\section{BITRATE RELATIONS USED BY THE NRIA}

In this section, we define some simple but usable bitrate relations in order to describe part of the problem with the NRIA that we aim to solve in Section 5.

The NRIA uses a predefined asymmetry parameter $a$ that specifies the ratio between the total desired downstream and upstream bitrates

$$
a=\frac{\sum_{u=1}^{U} R_{u, \mathrm{DS}}}{\sum_{u=1}^{U} R_{u, \mathrm{US}}} .
$$

Two "special cases" arise when $a=0$ and $a=\infty$. For $a=0$, the total cable capacity is assigned to the upstream transmission direction; thus, we transmit only in the upstream.
TABLE 1: Example: relations between different bitrates and users' priority values.

\begin{tabular}{ccccccc}
\hline User & \multicolumn{2}{c}{ User priorities } & \multicolumn{2}{c}{ User bitrates } & \multicolumn{2}{c}{ Norm. bitrates } \\
$u$ & $\alpha_{u, \mathrm{DS}}$ & $\alpha_{u, \mathrm{US}}$ & $R_{u, \mathrm{DS}}$ & $R_{u, \mathrm{US}}$ & $R_{u, \mathrm{DS}} / \alpha_{u, \mathrm{DS}}$ & $R_{u, \mathrm{US}} / \alpha_{u, \mathrm{US}}$ \\
\hline 1 & $1 / 3$ & $1 / 6$ & 4 & 1 & 12 & 6 \\
2 & $2 / 3$ & $5 / 6$ & 8 & 5 & 12 & 6 \\
$\Sigma$ & 1 & 1 & 12 & 6 & - & - \\
\hline
\end{tabular}

For $a=\infty$, the total cable capacity is assigned to the downstream transmission direction; thus, we transmit only in the downstream.

For a given transmission direction dir, with dir $\in\{\mathrm{DS}$, US\}, we do not know a priori which bitrates can be supported by each user. Therefore, we assign to each user a priority value $\alpha_{u \text {,ir }}$, which specifies how much of the total cable capacity (in a certain transmission direction) will be assigned to user $u$. Hence, we specify the relation between the user priorities and the user bitrates as

$$
\frac{R_{1, \mathrm{dir}}}{\alpha_{1, \mathrm{dir}}}=\frac{R_{2, \mathrm{dir}}}{\alpha_{2, \mathrm{dir}}}=\cdots=\frac{R_{U, \mathrm{dir}}}{\alpha_{U, \mathrm{dir}}},
$$

with

$$
\sum_{u=1}^{U} \alpha_{u, \mathrm{dir}}=1
$$

A "special case" arises when $\alpha_{u \text {,dir }}=0$. In this case, the user $u$ is not transmitting in the particular direction dir; thus, it is removed from (7).

It can be shown that the downstream and upstream bitrates for each user are related by

$$
R_{u, \mathrm{DS}}=a \cdot \frac{\alpha_{u, \mathrm{DS}}}{\alpha_{u, \mathrm{US}}} \cdot R_{u, \mathrm{US}}, \quad \text { for } u=1,2, \ldots, U .
$$

Let us illustrate these parameters with a hypothetic but simple two-user example, consisting of one private user and one business user. First, let us assume that the two-pair cable has a total capacity of $18 \mathrm{Mbps}$ (which is in reality unknown), and let us assume that we have a business model that specifies the asymmetry $a=2$ between the downstream and upstream directions. From the formulas above, we now have $R_{1, \mathrm{DS}}+R_{2, \mathrm{DS}}=12$ and $R_{1, \mathrm{US}}+R_{2, \mathrm{US}}=6$. Next, let us assign the downstream $\alpha_{1, \mathrm{DS}}=1 / 3$ to the private user and $\alpha_{2 \text {,DS }}=2 / 3$ to the business user, which gives downstream rates of $4 \mathrm{Mbps}$ and $8 \mathrm{Mbps}$ to the private user and to the business user, respectively.

Similarly, in the upstream let us assign $\alpha_{1, \mathrm{US}}=1 / 6$ (private) and $\alpha_{2, \mathrm{US}}=5 / 6$ (business), which gives $1 \mathrm{Mbps}$ $\left(=R_{1, \mathrm{US}}\right)$ and $5 \mathrm{Mbps}\left(=R_{2, \mathrm{US}}\right)$ to the private user and to the business user, respectively. It can easily be verified that the users' bitrates and priority values fulfill (7) and (8), respectively. Table 1 summarizes the different parameter values given in this example.

In an actual network scenario, we do not know beforehand which bitrates can be supported. However, the NRIA uses the given asymmetry parameter $a$ and the user priority values $\alpha_{u \text {,dir }}$ to find the desired operating point, (i.e., bitrates of all users) since the quantities represented by these parameters are always related through (6), (7), and (8). 


\section{PROBLEM FORMULATION}

The IWFA and the OSBA assume a fixed D-FDD bandplan. This makes it difficult to balance the bitrates between the downstream and upstream transmission directions. As a result many times in a particular transmission direction much higher bitrates are achieved than those we want to offer and at the same time in the other direction we cannot offer the desired bitrates. Furthermore, in a particular direction, the IWFA assumes that the target bitrates of all users are known a priori and that they are achievable. On the other hand the OSBA uses some form of exhaustive search, which is time consuming, to find the desired operation point. Therefore, with these problems in mind we take a different approach with the NRIA.

As mentioned earlier, the NRIA aims to jointly optimize a bandplan and the power allocation for all users. That is, the NRIA selects the downstream and upstream subcarriers common for all users represented by the sets $I_{\mathrm{DS}}$ and $I_{\mathrm{US}}$, with $I_{\mathrm{DS}} \cap I_{\mathrm{US}}=\varnothing$. Hence, the users' downstream and upstream bitrates will depend on each other, a property that is often desirable for practical business models. The dependency between the downstream and upstream bitrates guides the NRIA to desirable operating points, as in the example given in Section 3. Furthermore, to jointly optimize the power allocation among all users, two vectors are to be found for each user, specifying the power allocation in the downstream direction, $\mathcal{P}_{u, \mathrm{DS}}=\left[\mathcal{P}_{u, \mathrm{DS}}^{0}, \mathcal{P}_{u, \mathrm{DS}}^{1}, \ldots, \mathcal{P}_{u, \mathrm{DS}}^{N-1}\right]$, and in the upstream direction, $\mathcal{P}_{u, \mathrm{US}}=\left[\mathcal{P}_{u, \mathrm{US}}^{0}, \mathcal{P}_{u, \mathrm{US}}^{1}, \ldots, \mathcal{P}_{u, \mathrm{US}}^{N-1}\right]$. In addition, each user should satisfy total power constraints: $0 \leq \sum_{n} \mathcal{P}_{u, \mathrm{DS}}^{n} \leq \mathcal{P}_{u, \mathrm{DS}}^{\max }$ and $0 \leq \sum_{n} \mathcal{P}_{u, \mathrm{US}}^{n} \leq \mathcal{P}_{u, \mathrm{US}}^{\max }$, where $\mathcal{P}_{u, \mathrm{DS}}^{\max }$ and $\mathcal{P}_{u, \mathrm{US}}$ denote the maximum total power allowed for user $u$ in the downstream and upstream directions, respectively. Usually the maximum total power constraint is selected the same for all users.

We aim to jointly maximize the bitrates in the downstream and in the upstream for all users under the constraints that the bitrates should satisfy the predefined relations (6), (7), and (8). Without these constraints a search for a maximized total bitrate would lead to a situation where the users close to the $\mathrm{CO}$ (or cabinet) being given very high bitrates at the price of the distant users, who will get very low bitrates or no DSL service at all.

When formulating the optimization problem, it is convenient to use two indicators for each subcarrier, $\beta_{\mathrm{DS}}^{n}$ and $\beta_{\mathrm{US}}^{n}$, which specify the transmission direction. Due to the D-FDD Zipper type transmission scheme, ${ }^{2}$ the subcarrier indicators fulfill $\beta_{\mathrm{DS}}^{n}=1-\beta_{\mathrm{US}}^{n}$ for $n=0,1, \ldots, N-1$. For the upstream transmission direction, the relation between $\beta_{\mathrm{US}}$ and $I_{\mathrm{US}}$ is given by

$$
\beta_{\mathrm{US}}^{n}= \begin{cases}1, & \text { if } n \in I_{\mathrm{US}} \\ 0, & \text { otherwise }\end{cases}
$$

\footnotetext{
${ }^{2}$ Without loss of generality, we do not consider the silent (unused) subcarriers.
}

A corresponding relation holds for the downstream direction. Using these indicators, (2) can be written as

$$
R_{u, \mathrm{US}}=\sum_{n \in I_{\mathrm{US}}} R_{u}^{n}=\sum_{n=0}^{N-1} \beta_{\mathrm{US}}^{n} R_{u}^{n} .
$$

The optimization problem can now be formulated as follows:

$$
\begin{gathered}
\text { maximize } \sum_{u=1}^{U}\left(R_{u, \mathrm{DS}}+R_{u, \mathrm{US}}\right), \\
\text { subject to } \sum_{u=1}^{U} R_{u, \mathrm{DS}}=a \sum_{u=1}^{U} R_{u, \mathrm{US}}, \\
\frac{R_{1, \mathrm{DS}}}{\alpha_{1, \mathrm{DS}}}=\frac{R_{2, \mathrm{DS}}}{\alpha_{2, \mathrm{DS}}}=\cdots=\frac{R_{U, \mathrm{DS}}}{\alpha_{U, \mathrm{DS}}}, \\
\frac{R_{1, \mathrm{US}}}{\alpha_{1, \mathrm{US}}}=\frac{R^{2, \mathrm{US}}}{\alpha_{2, \mathrm{US}}}=\cdots=\frac{R^{U, \mathrm{US}}}{\alpha_{U, \mathrm{US}}}, \\
\sum_{u=1}^{U} \alpha_{u, \mathrm{DS}}=1, \quad \sum_{u=1}^{U} \alpha_{u, \mathrm{US}}=1, \\
\sum_{n=0}^{N-1} \beta_{\mathrm{DS}}^{n} \mathcal{P}_{u, \mathrm{DS}}^{n} \leq \mathcal{P}_{u, \mathrm{DS}}^{\max }, \quad u=1,2, \ldots, U, \\
\sum_{n=0}^{N-1} \beta_{\mathrm{US}}^{n} \mathcal{P}_{u, \mathrm{US}}^{n} \leq \mathcal{P}_{u, \mathrm{US}}^{\max }, \quad u=1,2, \ldots, U, \\
\beta_{\mathrm{DS}}^{n}=1-\beta_{\mathrm{US}}^{n}, \quad n=0,1, \ldots, N-1, \\
\beta_{\mathrm{DS}}^{n}, \beta_{\mathrm{US}}^{n} \in\{0,1\}, \\
\mathcal{P}_{u, \mathrm{DS}}^{n}, \mathcal{P}_{u, \mathrm{US}}^{n} \in\left[0, \mathbb{R}^{+}\right] .
\end{gathered}
$$

The asymmetry parameter $a$, and the user priority values $\alpha$, are all constants (and a designer's choice) for the NRIA, as explained in Section 3. A PSD constraint is often given for practical implementations of DSL modems. If this is the case the allowed power range $\left[0, \mathbb{R}^{+}\right]$, where $\mathbb{R}^{+}$denotes the real positive numbers, in $(12 \mathrm{j})$ should be replaced with $\left[0, \ldots, \mathcal{P}_{u}^{n, \text { max }}\right]$, where $\mathcal{P}_{u}^{n, \text { max }}$ denotes the maximum PSD level allowed for user $u$ on subcarrier $n$ for a given transmission direction. In practice, the PSD mask constraint is usually the same for all DSL systems of the same type.

The optimization problem (12) involves binary variables from (12i) and continuous variables for the PSDs. Furthermore, we have nonlinear relations between binary and continuous variables in the optimization (12a) as well as in the constraints (12b), (12c), and (12d), which are related also through (11). Therefore, (12) is a mixed-integer nonlinear optimization problem [12], which in general is very challenging from a computational point of view. Even for a fixed downstream and upstream subcarrier allocation, the objective function (12) and the constraints (12b), (12c), and (12d) are neither convex nor concave with respect to the users' power allocations. Thus, this type of optimization problem is not solvable with existing algorithms $[12,13]$.

In theory, it is possible to exhaustively try out all possible combinations of subcarrier allocations and, for each allocation, to try all possible combinations of PSD mask realizations for all users. However, the number of combinations is 
tremendously high and practically infeasible. For instance, in VDSL Zipper-DMT [14-16] with 4096 subcarriers this results in $2^{4096}$ possible combinations of subcarrier allocations. The number of possible combinations of PSD mask realizations of all users for a particular transmission direction is $\left(R^{n, \max }+1\right)^{N_{\text {dir }} \cdot U}$, where $R^{n, \max }$ denotes the maximum number of bits that can be loaded on a subcarrier, and $N_{\text {dir }}$ is the number of subcarriers used in a particular transmission direction. Thus, a rather typical case with $R^{n, \max }=15$ bits, $U=10$ users, and $N_{\text {dir }}=2048$ upstream subcarriers has $16^{20480}$ possible PSD mask realizations.

For a similar optimization problem but with a fixed subcarrier allocation, a dual decomposition method has been suggested [5-7]. In particular, the OSBA has reduced the search space for possible PSD mask realizations and has linear complexity in the number of subcarriers $N_{\text {dir }}$. However, the OSBA still has a complexity that increases exponentially with the number of users $U$ making it too complex for most of the DSL access network scenarios that are found in practice.

In the following section, we propose the normalized-rate iterative algorithm that solves the formulated optimization problem in a suboptimal way.

\section{THE NORMALIZED-RATE ITERATIVE ALGORITHM}

The normalized-rate iterative algorithm (NRIA) consists of two levels of nested iterations: an outer stage that searches for an optimized downstream and upstream subcarrier allocation; and an inner stage, which calculates the downstream and upstream bitrates that can be supported by each user. The inner stage is performed independently for each transmission direction, since D-FDD is considered.

In the outer stage, to make the bandplan optimization tractable, the NRIA divides the subcarriers into a small number of subbands with an equal number of subcarriers per subband. The subbands are assigned to the downstream and upstream directions in alternating order (cf. Section 5.1 "initial bandplan"). Then, depending on the downstream and upstream supported bitrates in the inner stage, the subband edges (and with that also the downstream and upstream subcarrier allocation) are adapted so that the desired asymmetry $a$ is achieved. This is described in more detail in Section 5.1 "change bandplan."

In the inner stage, a modified version of the fixed-margin water-filling (FM-WF) algorithm is deployed, which implicitly performs power control and power allocation, (cf. Section 5.1 "modified FM-WF algorithm"). The power control is achieved by constraining the users to only utilize the power needed to support the bitrates that satisfy the relations defined in (7) and to jointly maximize the bitrates of all users.

In the inner stage, the algorithm iterates over all users, which are ordered as described in Section 5.1 "user ordering," and derives a new target bitrate for each iteration. To achieve this, the NRIA uses the normalized supported bitrates $\bar{R}_{u \text {,dir }}(i)=R_{u \text {,dir }}(i) / \alpha_{u \text {,dir }}$, obtained in the last iterations. The target bitrate $T_{u \text {,ir }}(i+1)$ is the bitrate that the NRIA aims to achieve for user $u$ in the next iteration. Because it

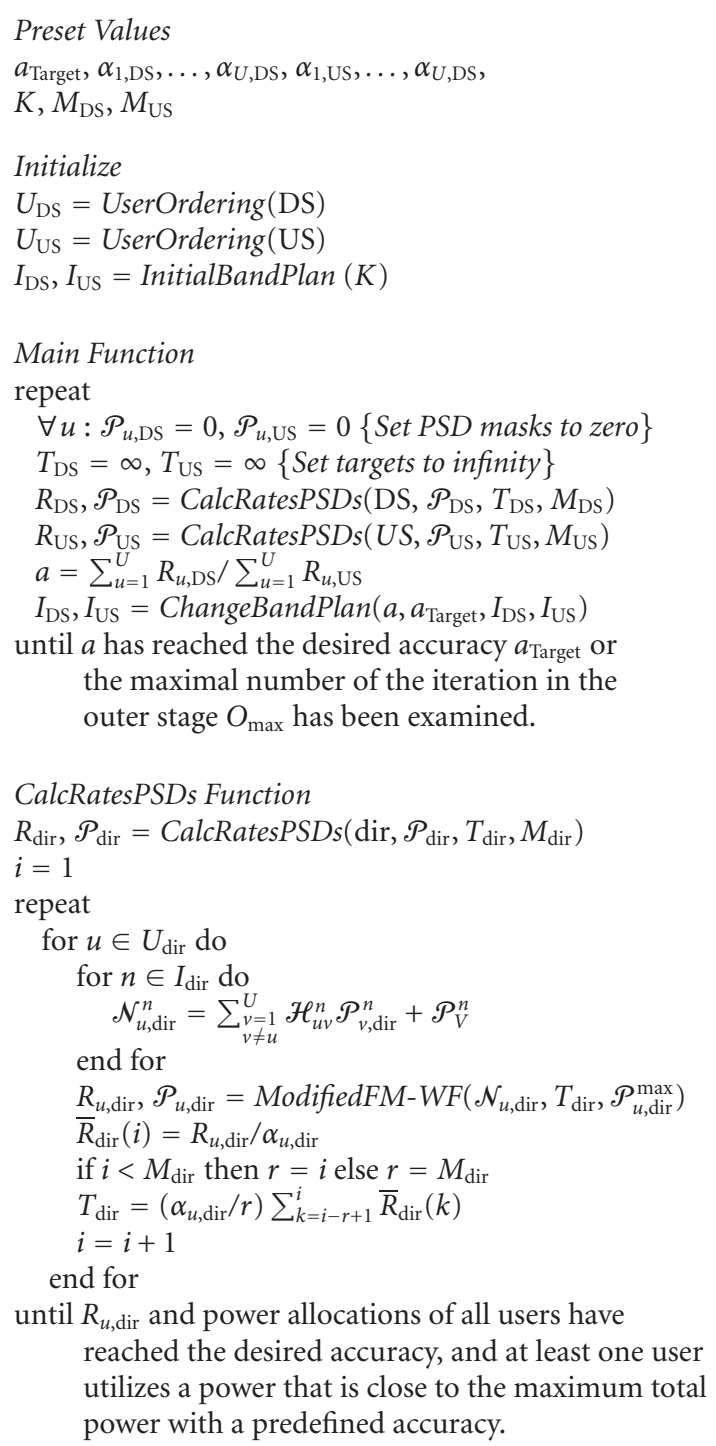

Algorithm 1: The normalized-rate iterative algorithm.

is difficult to make any probabilistic assumptions about any truly achievable bitrates with the given constraints and degrees of freedom, the NRIA makes a simple ad hoc estimate, which is the average of some past normalized achieved bitrates,

$$
T_{u, \operatorname{dir}}(i+1)=\frac{\alpha_{u, \mathrm{dir}}}{M} \sum_{m=0}^{M-1} \bar{R}_{\mathrm{dir}}(i-m) .
$$

The appropriate memory $M$ is somewhat related to the number of users, for example, the NRIA works well with $M=U$ [1].

The pseudocode of the NRIA is listed as Algorithm 1. The outer stage of the NRIA is the Main Function and the inner stage the CalcRatesPSDs Function. A PSD mask constraint can simply be incorporated into the algorithm in the 


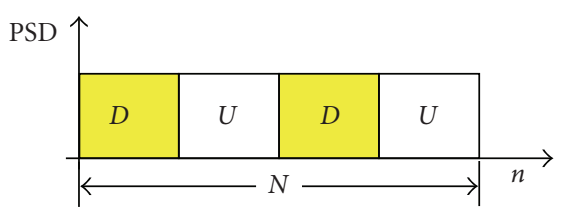

FIgURE 2: An example of initial subcarrier allocation, where the total number of available subcarriers $N$ are divided into $K=4$ subbands.

function ModifiedFM-WF as it is an inherent property of any type of water-filling (bit-loading) algorithm.

\subsection{Algorithmic details}

User ordering. Due to the estimation of the target bitrate in each iteration of the inner stage, the user ordering over which the NRIA iterates become important for the convergence speed of the algorithm. To speed up convergence, the users should first be arranged in decreasing priority order and the users within the same priority group should be arranged in order of decreasing line attenuation. The NRIA performs this ordering independently for both transmission directions. An extensive analysis with objectives and requirements for such an ordering is given in [3].

Initial bandplan. The NRIA partitions the available spectrum into $K$ subbands, with $N / K$ subcarriers in each. In order to simplify the description but without loss of generality, we assume that both $N$ and $K$ are powers of two. When there are some unused or silent subcarriers, they are simply zeroed in the algorithm and kept outside the optimization process.

An example with four subbands is shown in Figure 2. It is also possible to start with an upstream subband in the low frequencies. However, in practice, we usually start with a downstream subband to be spectrally compatible with asymmetric DSL (ADSL) downstream transmission.

Change bandplan. For a given subcarrier allocation, the inner stage of the NRIA calculates the bitrates for all users in the downstream and upstream directions. Then, depending on the achieved bitrates of all users and the predefined asymmetry $a$, the NRIA performs a binary search within the subbands for a new subcarrier allocation. This is performed with the constraint given in (12b). There are three cases:

(c1) $\sum_{u=1}^{U} R_{u, \mathrm{DS}}>a \sum_{u=1}^{U} R_{u, \mathrm{US}}$, which indicates that more subcarriers should be assigned in the upstream direction,

(c2) $\sum_{u=1}^{U} R_{u, \mathrm{DS}}<a \sum_{u=1}^{U} R_{u, \mathrm{US}}$, which indicates that more subcarriers should be assigned in the downstream direction, and

(c3) $\sum_{u=1}^{U} R_{u, \mathrm{DS}}=a \sum_{u=1}^{U} R_{u, \mathrm{US}}$, which indicates that an optimized subcarrier allocation has been found and that the outer stage, and also the whole NRIA, is complete.

A binary search within the subbands for the new downstream and upstream subcarrier allocation is performed, depending on the criteria (c1) and (c2), by simultaneously moving all right-hand downstream subband edges dotted with bullets

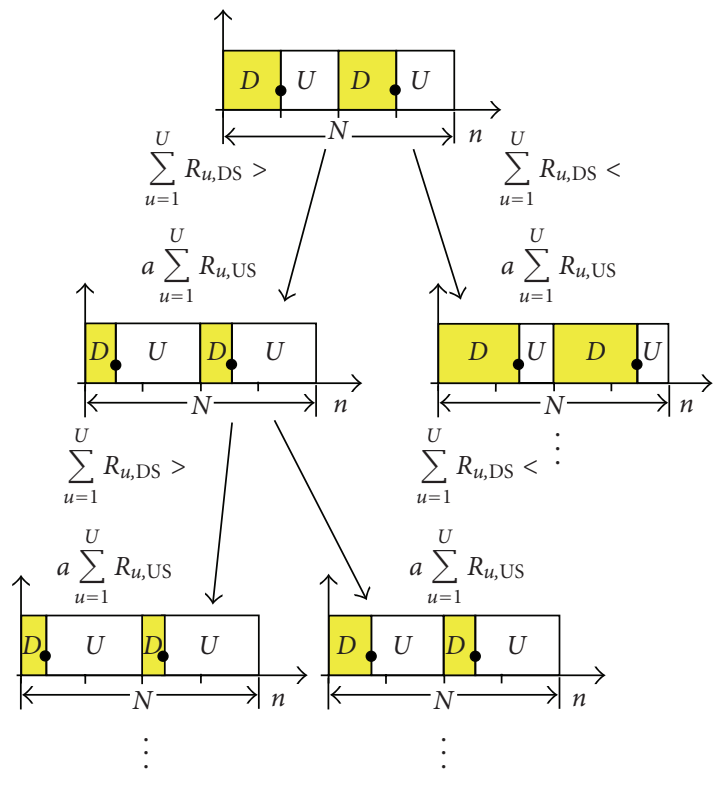

FIGURE 3: Illustration of the search for a subcarrier allocation.

to the right or to the left as shown in Figure 3. The subcarrier allocation is the same for all users, since FDD is considered.

The number of iterations in the outer stage depends on the number of subcarriers per subband and on the predefined accuracy with which the inequalities (c1) and (c2) should be fulfilled. However, the maximum number of iterations $O_{\max }$ in the outer stage can always be determined in advance, and this depends only on the number of subcarriers per subband, $N / K$. Therefore, due to the binary search, $O_{\max }=\log _{2}(N / K)+1$. For example, when the number of subcarriers is $N=4096$, as in VDSL, and when we select $K=8$ subbands, then $O_{\max }=\log _{2}(512)+1=10$.

Modified fixed-margin water-filling (FM-WF) algorithm. The water-filling (bit-loading) algorithm used in the inner stage is a modified version of the FM-WF algorithm [17]. The FM-WF algorithm uses only the power needed to achieve a predefined target bitrate. As described, we do not know a priori if a specific target bitrate can be supported for a given maximum total power. Therefore, we have modified the fixed-margin water-filling algorithm as follows: if the target bitrate can be supported, then only the power needed to support that bitrate is used; otherwise, the maximum total power is used and the supported bitrate is calculated.

The pseudocode of the modified FM-WF algorithm, when the continuous bit-loading is used as a water-filling algorithm, is given in [3].

Check the convergence point. One solution to find the downstream and upstream bitrates that maximize (12a) while satisfying the defined bitrate relations is to exhaustively test all possible maximum total power constraints among the users and to select the appropriate bitrates. Due to the number of combinations that we have to test this is in practice unfeasible. However, since the NRIA estimates target bitrates for each user, it can converge to a point where the sum of the users' bitrates is lower than can actually be 
achieved compared to exhaustive search. Fortunately, by using Postulate 1 below, such cases can always be detected and an improved estimate of the corresponding target bitrates can be derived.

Postulate 1. Consider a multiuser D-FDD transmission system operating in an interference channel where each receiver considers the crosstalk signal as noise. For such a multiuser system the sum of the user bitrates increases when the power of each user increases.

In Appendix A, we discuss the validly of this Postulate and prove it for a special case. Postulate 1 shows that the NRIA has converged to a "wrong" point if none of the modems has utilized the maximum total power after finishing the inner stage. For these cases, a performance improvement is achieved if the last $M$ values of normalized supported bitrates $\bar{R}$ are increased by some amount $\Delta \bar{R}$, and we continue iterating in the inner stage.

We propose to change $\Delta \bar{R}$ adaptively, depending on the maximum total power $P^{\max }$, and the maximum power used by any user $P^{\text {used }}$. Due to our definition in (7), at the convergence point, all the last $M$ normalized bitrates are approximately equal. Thus, we propose to increase the $\Delta \bar{R}$ by

$$
\Delta \bar{R}=\lambda \cdot \frac{P^{\max }-P^{\mathrm{used}}}{P^{\max }} \cdot \bar{R},
$$

where $\bar{R}$ is any one of the last $M$ normalized supported bitrates and $\lambda$ is a scaling factor.

Figure 4 shows the number of corrections in the estimate of the target bitrate $T_{\mathrm{US}}$, for different values of the scaling factor $\lambda$. The values of $T_{\text {US }}$ and $\lambda$ are shown for the case when $P^{\max }$ and $P^{\text {used }}$ are given in $\mathrm{dBm}$. The simulations were performed with the target bitrate $T_{\text {US }}$ initialized to $400 \mathrm{kbps}$ and for the network scenario shown in Figure 5, where the length of the first and second loops are $600 \mathrm{~m}$ and $1200 \mathrm{~m}$, respectively. During the simulation we have assumed that both users have equal priorities; thus, $\alpha_{1, \mathrm{US}}=\alpha_{2, \mathrm{US}}=0.5$.

The number of corrections in the estimate of the target bitrates in Figure 4 is high for low values of $\lambda$. However, when the NRIA converges to a wrong point, it will already be in the upper part of the curves shown in Figure 4. For $P^{\max }$ and $P^{\text {used }}$ given in $\mathrm{dBm}$, the NRIA is found to work well when $0<\lambda \leq 0.05$.

\section{SIMULATION RESULTS AND DISCUSSIONS}

All simulations in this paper are based on a Zipper-DMT type VDSL system [14-16], because this is the only standardized DSL technology which supports D-FDD transmission. There is little or no flexibility to change the standardized VDSL bandplans, but they can easily be changed to be much more flexible since D-FDD is an inherent property in Zipper duplexing.

We compare the performance of the NRIA with the other proposed algorithms for DSM: the IWFA, the OSBA, and the bi-IWFA. In order to do a fair comparison between the NRIA and the OSBA we use the Levin-Campello [18] water-filling

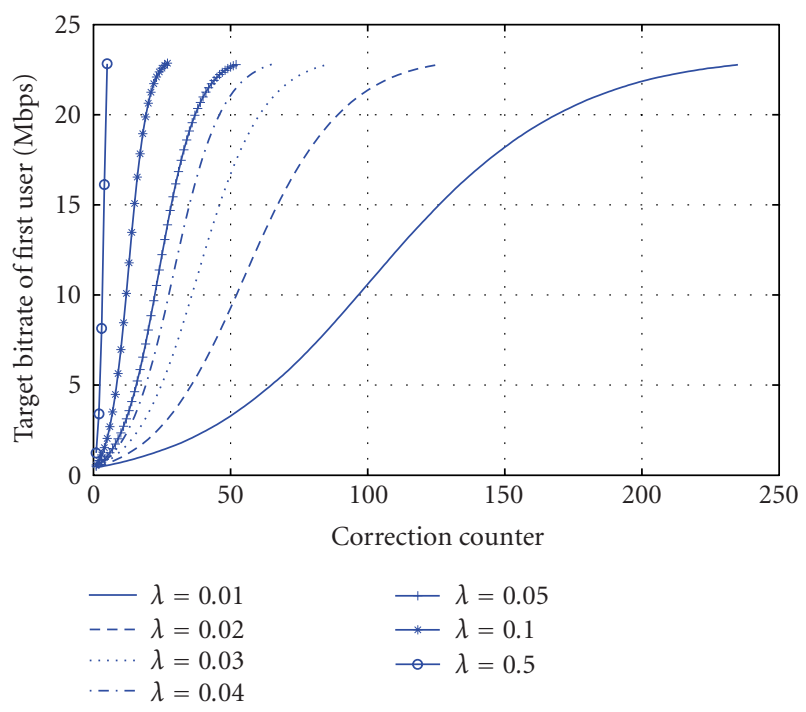

FIgURE 4: Example of the number of target bitrate corrections for a user that is initialized at $400 \mathrm{kbps}$.

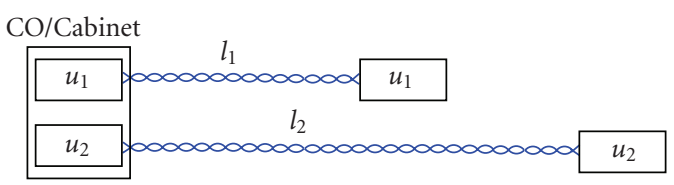

FIgURE 5: Network scenario used for most of the simulations.

algorithm to ensure integer bit-loading with maximum 15 bits per subcarrier. We also use Levin-Campello waterfilling to compare the NRIA with the IWFA. However, to compare the performance between the NRIA and the biIWFA we will use "continuous" bit-loading, that is, normal water-filling, for easier interpretations of the power allocations in the bi-IWFA.

The maximum total power for each user and each transmission direction is set to $11.5 \mathrm{dBm}$. The center frequency separation between two successive subcarriers is $4.3125 \mathrm{kHz}$ and the DMT symbol rate is $4 \mathrm{kHz}$, as specified in the VDSL DMT standards [14-16]. Furthermore, we use a DMT system with 4096 subcarriers but only the subcarriers in the range from 32 to 2782 are used, which corresponds to frequencies from $138 \mathrm{kHz}$ to $12 \mathrm{MHz}$. Moreover, for all simulations we have also included alien noise according to ETSI VDSL "noise model A" [14] in addition to the background noise. The FEXT and NEXT models are also specified in [14]. The used cable is the so called "BT_dwug" [19], which has $0.5 \mathrm{~mm}$ conductors. For most of the simulations we will use a two-user scenario $U=2$, as shown in Figure 5. This is essentially the same network scenario as used in [5], however, we have not collocated four modems at each node. This is because the IWFA, due to the iterative process, might generate different PSDs to support equal bitrates for the collocated modems although they have the same FEXT couplings. 
To achieve a bit error rate of $10^{-7}$ we have assumed an SNR gap, $\Gamma=12.3 \mathrm{~dB}$. It is derived as $\Gamma=\Gamma_{\text {Mod }}+\Gamma_{\text {Noise }}-\Gamma_{\text {Code }}$ $=9.8+6-3.5=12.3 \mathrm{~dB}$, where $\Gamma_{\text {Mod }}$ denotes the modulation gap, which for quadrature amplitude modulation signal constellation is $9.8 \mathrm{~dB}$ [17]; $\Gamma_{\text {Noise }}$ denotes the noise margin, which is assumed to be $6 \mathrm{~dB}$; and $\Gamma_{\text {Code }}$ denotes the coding gain, which is set to $3.5 \mathrm{~dB}$.

$K=8$ downstream and upstream subbands are typically sufficient to achieve the desirable bitrates [1], which is also used in this paper. As suggested in Section 5, we select $M_{\mathrm{DS}}=$ $M_{\mathrm{US}}=U$.

\subsection{Rate regions}

To compare the performance of the NRIA with the IWFA and the OSBA we will use the rate region concept, which characterizes all possible bitrate combinations among the users. Due to the fixed subcarrier allocation, the downstream and upstream rate regions of the IWFA and the OSBA are independent and $U$-dimensional. For example, in a two-user case the rate regions of the IWFA and the OSBA can be plotted in a two-dimensional space, as shown in Figure 6, and any pair of bitrates can independently be selected from the downstream and upstream rate regions. Furthermore, any pair of bitrates that lies inside the rate regions can be supported by the IWFA. With OSBA, however, any pair of bitrates that lie on the rate region boundaries can be found, because only this pair of bitrates maximizes the weighted sum of the bitrates [6].

Since the NRIA searches for an optimized downstream and upstream subcarrier allocation the downstream and upstream rate regions becomes dependent. For a two-user case the NRIA finds two pairs of downstream and upstream bitrates $\left(R_{1, \mathrm{DS}}, R_{2, \mathrm{DS}}\right)$ and $\left(R_{1, \mathrm{US}}, R_{2, \mathrm{US}}\right)$, which are related by three independent parameters; $a, \alpha_{1, \mathrm{DS}}$, and $\alpha_{1, \mathrm{US}}$ as described in Section 3. Thus, the NRIA supported rate regions for the two-user case are five-dimensional. This is difficult to visualize and to compare with the two-dimensional rate regions of the IWFA and the OSBA. Furthermore, the NRIA finds only those pairs of bitrates that lie on the rate regions boundaries, because only those pairs maximize the sum of downstream and upstream bitrates and satisfy the relations defined in (9).

One way to compare the NRIA with the IWFA and the OSBA is to calculate the parameters needed by the NRIA from the two-dimensional rate regions spanned by the IWFA and the OSBA. For example, let us select the pair of bitrates at point $A$ for the downstream and the pair of bitrates at point $B$ for the upstream as shown in Figure 6. For these two pairs of bitrates we can calculate the asymmetry parameter $a$ and the users' priority values $\alpha_{u \text {,DS }}$ and $\alpha_{u}$,Us, needed in NRIA by using (6) and (7). We can repeat this for any two pairs of downstream and upstream bitrates and draw the corresponding downstream and upstream rate regions of the NRIA. However, this strategy excludes a large portion of the bitrates that are supported by the NRIA but not by the IWFA and the OSBA. Hence, such a comparison would therefore become quite skewed.

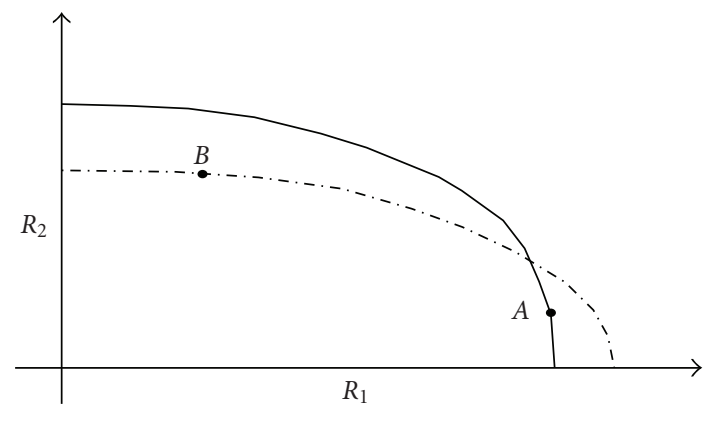

- DS
$\cdots-$ US

Figure 6: Illustration of rate regions of the IWFA and OSBA.

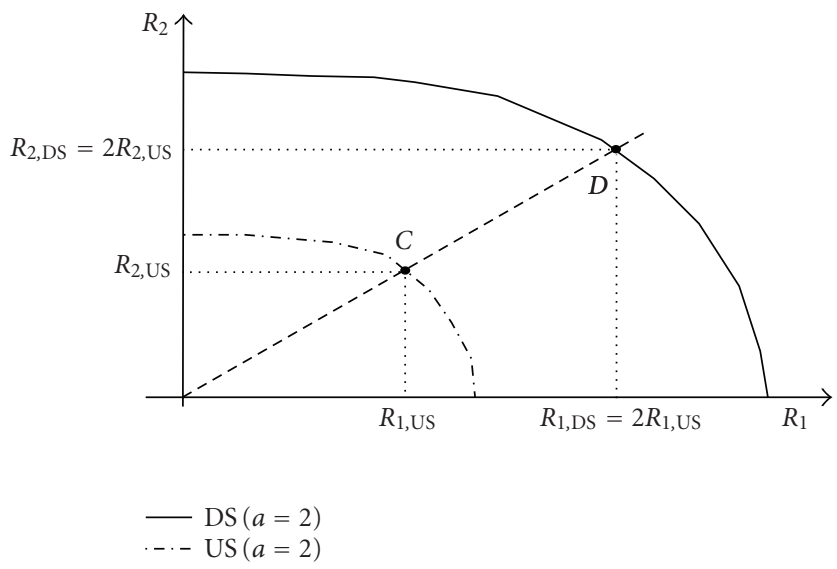

FIGURE 7: An example of rate regions of the NRIA for two-user case when $\alpha_{1, \mathrm{DS}}=\alpha_{1, \mathrm{US}}$ and $\alpha_{2, \mathrm{DS}}=\alpha_{2, \mathrm{US}}$, and for asymmetry parameter value, $a=2$.

Instead, we will use another way to compare the three algorithms. We will assume equal downstream and upstream user priorities for the NRIA, which for the two-user case from (9) yields

$$
R_{u, \mathrm{DS}}=a R_{u, \mathrm{US}}, \quad \text { for } u=1,2 .
$$

Under this assumption, for a fixed asymmetry parameter value $a$, we can plot the rate regions of the NRIA in twodimensional space. However, note that the NRIA will now only support those downstream and upstream bitrates for which $\alpha_{u, \mathrm{US}}=\alpha_{u \text {,DS}}$. Thus, we calculate two pairs of downstream and upstream bitrates $\left(R_{1, \mathrm{US}}, R_{2, \mathrm{US}}\right)$ and $\left(R_{1, \mathrm{DS}}, R_{2, \mathrm{DS}}\right)=\left(a R_{1, \mathrm{US}}, a R_{2, \mathrm{US}}\right)$, respectively. As a result, depending on $a$, the downstream bitrates are either expanded or contracted compared to the upstream bitrates. That is, two pairs of downstream and upstream bitrates lie on a line that also crosses the origin of the bitrate axes. This line will be included in some plots to better illustrate the bitrate relations between the different algorithms. 
Figure 7 shows an example of the rate regions of the NRIA for the asymmetry parameter value set to $a=2$. In the same plot a pair of downstream bitrates at point $C$ and a pair of upstream bitrates at point $D$, which lie on a line that also crosses the origin, are also shown. Note that for the symmetric case, with $a=1, C=D$ and the corresponding rate regions coincide.

\subsection{Comparison of the NRIA with the IWFA}

In this section, we compare the performance of the NRIA with the iterative water-filling algorithm (IWFA) [4]. The IWFA assumes a fixed frequency bandplan, therefore, for all simulations concerning the IWFA we will use one of the standardized frequency bandplans: the bandplan "997," without guard bands, with the corresponding downstream and upstream subcarriers

$$
\begin{aligned}
& I_{\mathrm{DS}}=\{32 \cdots 695,1183 \cdots 1634\}, \\
& I_{\mathrm{US}}=\{696 \cdots 1182,1635 \cdots 2782\} .
\end{aligned}
$$

Figure 8 shows the downstream and upstream rate regions for the IWFA and for the NRIA with $a=1$, that is, NRIA forces symmetric bitrates for each user. To compare the NRIA with the IWFA we need to find the corresponding symmetric bitrates for the IWFA. They are located where the boundaries of the downstream and upstream IWFA rate regions intersect.

The bitrate figures for the symmetric case are summarized in Table 2. We see that if we fix the bitrate of user $u_{1}$ to $53.35 \mathrm{Mbps}$, as achieved by the IWFA, the NRIA can increase the bitrate of $u_{2}$ from 10.36 to $12.80 \mathrm{Mbps}$ (point $A$, an increase of $23 \%$ ). If we instead fix the bitrate of $u_{2}$ at $10.36 \mathrm{Mbps}$ the NRIA can increase the bitrate of $u_{1}$ from 53.35 to $57.50 \mathrm{Mbps}$ (point $B$, an increase of $8 \%$ ). The gain is smaller for the latter case, since $u_{1}$ disturbs $u_{2}$ more than vice versa, due to the upstream "near-far" problem [4].

For distributed DSL access networks in general, decreasing one user's bitrate does not necessarily increase another user's bitrate correspondingly. Therefore, in a third comparison the users' bitrate relations of the NRIA and the IWFA are equal

$$
\left(\frac{R_{1}}{R_{2}}\right)_{\text {IWFA }}=\left(\frac{R_{1}}{R_{2}}\right)_{\text {NRIA }}=\frac{\alpha_{1}}{\alpha_{2}} .
$$

This is depicted in Figure 8 at point $C$, where the dashed line (corresponding to (17)) intersects with NRIA's rate region boundary. For this case, a total bitrate increase of about $6 \%$ is achieved with the NRIA compared to the IWFA.

For a fixed subcarrier allocation (when only the inner stage of the NRIA is used), the NRIA cannot outperform the IWFA since the inner stage of the NRIA is based on the IWFA. However, in this case the NRIA can be used to calculate the set of maximum achievable users' bitrates for the IWFA. The alternative, to calculate the sets of achievable bitrates in the IWFA by exhaustively testing all possible maximum total power constraints [4] requires much higher computational complexity.

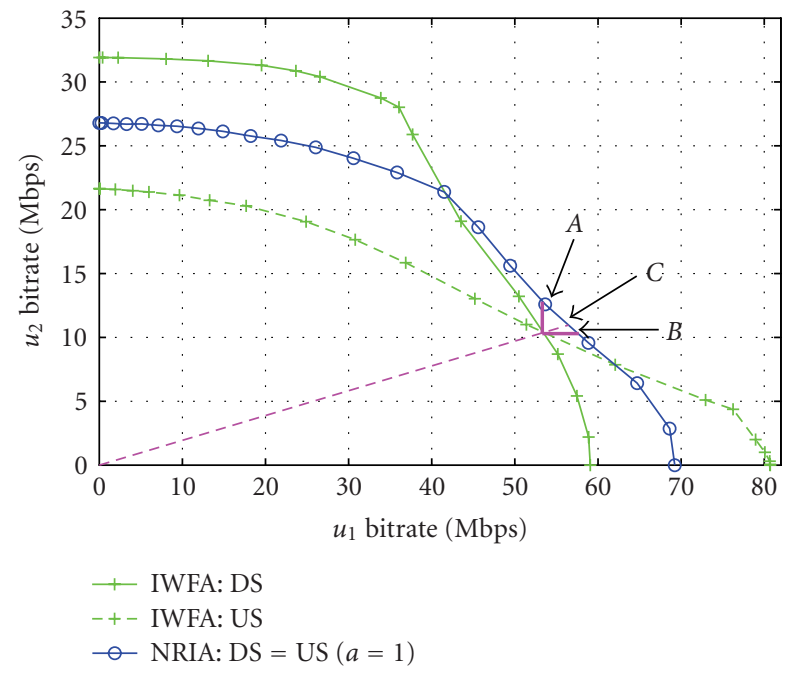

Figure 8: Downstream and upstream rate regions of IWFA and NRIA for $a=1, \alpha_{1, \mathrm{DS}}=\alpha_{1, \mathrm{US}}$, and $\alpha_{2, \mathrm{DS}}=\alpha_{2, \mathrm{US}}$.

TABLE 2: Comparison of the NRIA and the IWFA, symmetric bitrates $(a=1.00)$.

\begin{tabular}{lcccc}
\hline Algorithm & Direction & $\begin{array}{c}\text { User } u_{1} \\
(\mathrm{Mbps})\end{array}$ & $\begin{array}{c}\text { User } u_{2} \\
(\mathrm{Mbps})\end{array}$ & $\begin{array}{c}\text { Increase } \\
(\%)\end{array}$ \\
\hline IWFA & DS/US & 53.35 & 10.36 & - \\
NRIA $(A)$ & DS/US & 53.35 & 12.80 & 23.5 \\
NRIA $(B)$ & DS/US & 57.50 & 10.36 & 7.78 \\
NRIA $(C)$ & DS/US & 56.46 & 10.96 & 5.82 \\
\hline
\end{tabular}

We also compare the performance of the NRIA with the IWFA when the asymmetry parameter value is $a=1.25$, that is, higher downstream than upstream bitrates. Again, we should compare the NRIA with the IWFA for which $a=1.25$. In Figure 9 the bitrates for this case are found at the intersections of the dashed line with the IWFA downstream and upstream rate region boundaries. These bitrates are summarized in Table 3; and it can be verified that they satisfy the priority relations:

$$
\begin{aligned}
& \left(\frac{R_{1, \mathrm{DS}}}{R_{2, \mathrm{DS}}}\right)_{\mathrm{IWFA}}=\left(\frac{R_{1, \mathrm{DS}}}{R_{2, \mathrm{DS}}}\right)_{\mathrm{NRIA}}=\frac{\alpha_{1, \mathrm{DS}}}{\alpha_{2, \mathrm{DS}}}, \\
& \left(\frac{R_{1, \mathrm{US}}}{R_{2, \mathrm{US}}}\right)_{\mathrm{IWFA}}=\left(\frac{R_{1, \mathrm{US}}}{R_{2, \mathrm{US}}}\right)_{\mathrm{NRIA}}=\frac{\alpha_{1, \mathrm{US}}}{\alpha_{2, \mathrm{US}}} .
\end{aligned}
$$

We see that the NRIA achieves an increase of more than $12 \%$ in each transmission direction. The corresponding downstream and upstream transmit PSDs of the IWFA and the NRIA when $a=1.25$ are shown in Figures 10 and 11 .

The NRIA optimized downstream and upstream subcarrier allocation for the analyzed two-user network scenario with $K=8$ subbands, $a=1.25$ asymmetry, and the bitrates given in Table 3 are

$$
\begin{aligned}
& I_{\mathrm{DS}}=\{32 \cdots 477,1025 \cdots 1501,2049 \cdots 2525\}, \\
& I_{\mathrm{US}}=\{478 \cdots 1024,1502 \cdots 2048,2526 \cdots 2782\} .
\end{aligned}
$$




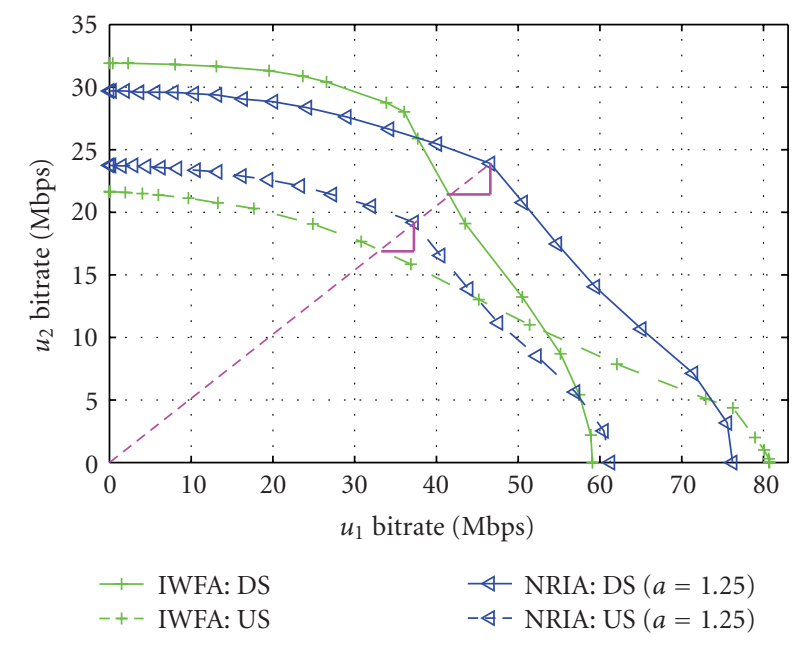

FIGURE 9: Downstream and upstream rate regions of the IWFA and NRIA for $a=1.25, \alpha_{1, \mathrm{DS}}=\alpha_{1, \mathrm{US}}$, and $\alpha_{2, \mathrm{DS}}=\alpha_{2, \mathrm{US}}$.

TABLE 3: Comparison of the NRIA and the IWFA, asymmetric bitrates $(a=1.25)$.

\begin{tabular}{lcccc}
\hline Algorithm & Direction & $\begin{array}{c}\text { User } u_{1} \\
(\mathrm{Mbps})\end{array}$ & $\begin{array}{c}\text { User } u_{2} \\
(\mathrm{Mbps})\end{array}$ & $\begin{array}{c}\text { Increase } \\
(\%)\end{array}$ \\
\hline IWFA & DS & 41.25 & 21.42 & - \\
IWFA & US & 33.25 & 16.88 & - \\
NRIA & DS & 46.60 & 23.88 & 12.4 \\
NRIA & US & 37.24 & 19.19 & 12.5 \\
\hline
\end{tabular}

Note that although we have selected eight subbands (four for each transmission direction), that has been reduced to six subbands (three for each transmission direction) since only subcarriers in the range $\{32, \ldots, 2782\}$ are used (out of 4096 total).

The transmit PSDs of the NRIA and the IWFA are nonsmooth due to the integer bit-loading algorithm. However, the PSDs of the NRIA and the IWFA are more or less flat over the used frequency spectrum. The PSDs of IWFA are almost flat as shown in $[20,21]$ when the integer bit-loading algorithm is used. Because the inner stage of the NRIA is based on the IWFA, the PSDs generated by the NRIA are likewise almost flat when the integer bit-loading algorithm is used.

The NRIA achieves better performance due to a better optimized bandplan and power allocations found by NRIA compared to the fixed bandplan and power allocations found by the IWFA. Let us analyze this for a particular case, which can be applied to other cases in a similar way. Consider the second user and the upstream transmission direction. From Figure 11 it can be shown that the NRIA and the IWFA utilize quite similar bandwidths and that the PSDs also have similar levels. However, compared to the IWFA, the NRIA utilizes low frequencies where the channel conditions typically are better (the noise-to-channel-gain ratio is low). This results in higher supported bitrates by the NRIA compared to the IWFA.

\subsection{Comparison of the NRIA with the OSBA}

In this section, we compare the NRIA with the optimal spectrum balancing algorithm (OSBA) and use the same two-user scenario as before (cf. Figure 5). Furthermore, as the OSBA assumes a fixed bandplan we use the same subcarrier allocation as used in Section 6.2 for the IWFA.

Figure 12 shows the downstream and upstream rate regions for the NRIA with symmetric bitrates $a=1$ and for the OSBA with varying asymmetry. The corresponding symmetric bitrates for the OSBA are given in Table 4. To allow user $u_{1}$ to have $52.32 \mathrm{Mbps}$ with the NRIA, as achieved by the OSBA, user $u_{2}$ can only have $13.55 \mathrm{Mbps}$ (point $A$, a loss of $33 \%$ ). Alternatively, to allow $u_{2}$ to have $20.13 \mathrm{Mbps}$ with the NRIA, as achieved by the OSBA, $u_{1}$ can have only $43.37 \mathrm{Mbps}$ (point $B$, a loss of $17 \%$ ). Finally, when we want to have the same bitrate relations with the NRIA as with the OSBA, then the bitrates given in Table 4 can be supported, which corresponds to a loss of $11 \%$ (point $C$ in Figure 12).

If we instead compare the performance of the OSBA with the NRIA for the asymmetry parameter value set to $a=1.25$ the downstream and upstream bitrates given in Table 5 can be supported. For this case the NRIA suffers a loss of less than $5 \%$ in both transmission directions.

These results are not surprising since the OSBA can in theory deliver the highest bitrates for a given bandplan. However, the OSBA has a much higher computational complexity compared to the NRIA. We have shown in [3] that when the Levin-Campello bit-loading algorithm is used, the computational complexity of the NRIA is

$$
\begin{aligned}
C_{\mathrm{NRIA}}= & \mathcal{O}\left(O \cdot i_{\mathrm{DS}} \cdot \tilde{N}_{\mathrm{DS}}(U+1)\right) \\
& +\mathcal{O}\left(O \cdot i_{\mathrm{US}} \cdot \tilde{N}_{\mathrm{US}}(U+1)\right),
\end{aligned}
$$

where $\mathcal{O}$ denotes the complexity order; $O$ denotes the number of iterations in the outer stage; $U$ denotes the number of users; $i_{\mathrm{DS}}$ and $i_{\mathrm{US}}$ denote the number of iterations in the downstream and upstream inner stages, respectively; $\tilde{N}_{\text {DS }}$ denotes the average number of subcarriers in downstream assigned over all downstream outer stage iterations; and $\tilde{N}_{\text {Us }}$ denotes the average number of subcarriers in the upstream assigned over all upstream outer stage iterations.

The complexity of the OSBA for a particular transmission direction is [6]

$$
C_{\mathrm{OSBA}}=\mathcal{O}\left(N_{\mathrm{dir}} U\left(R^{n, \max }+1\right)^{U} 33^{U}\right),
$$

where $N_{\text {dir }}$ denotes the number of subcarriers assigned in a particular transmission direction and $R^{n, \max }$ denotes the maximum number of bits that can be loaded on a subcarrier. For both transmission directions based on (21), the complexity of the OSBA is

$$
\begin{aligned}
C_{\mathrm{OSBA}}= & \mathcal{O}\left(N_{\mathrm{DS}} U\left(R^{n, \max }+1\right)^{U} 33^{U}\right) \\
& +\mathcal{O}\left(N_{\mathrm{US}} U\left(R^{n, \max }+1\right)^{U} 33^{U}\right) .
\end{aligned}
$$

First we analyze the complexity of the NRIA and the OSBA for the two-user case $(U=2)$. For the NRIA the maximum 


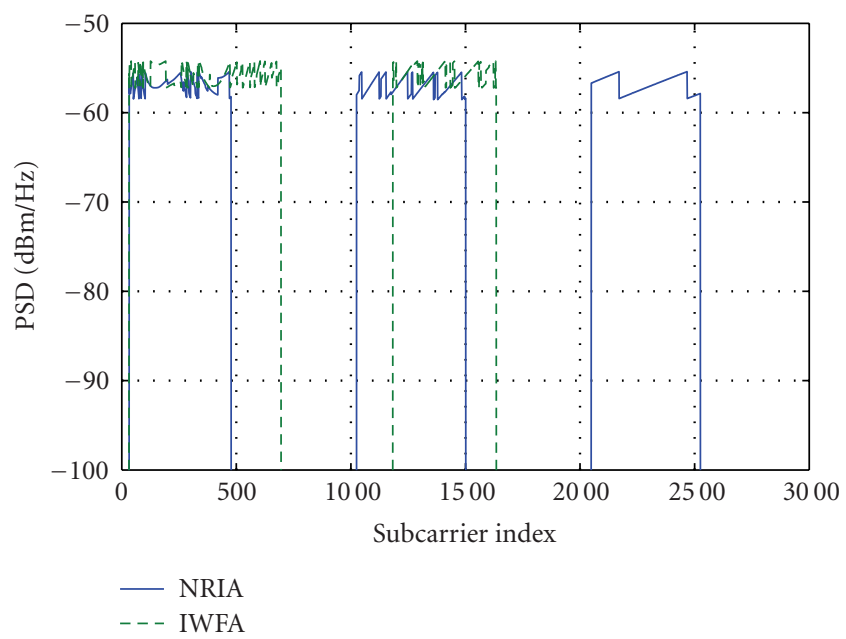

(a)

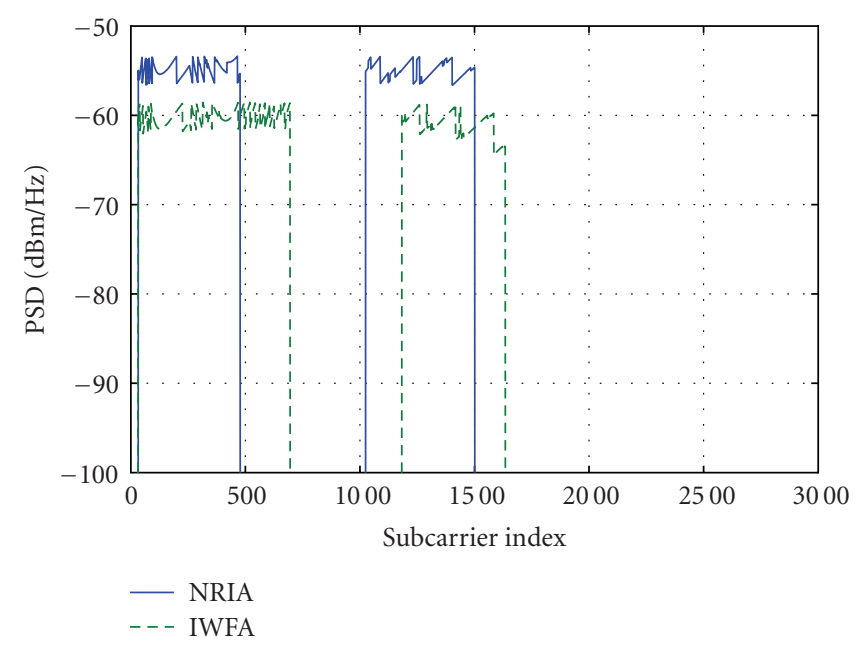

(b)

FIgure 10: Downstream transmit PSDs of the NRIA and the IWFA for users' bitrates given in Table 3. (a) $u_{1}$ downstream PSDs; (b) $u_{2}$ downstream PSDs.

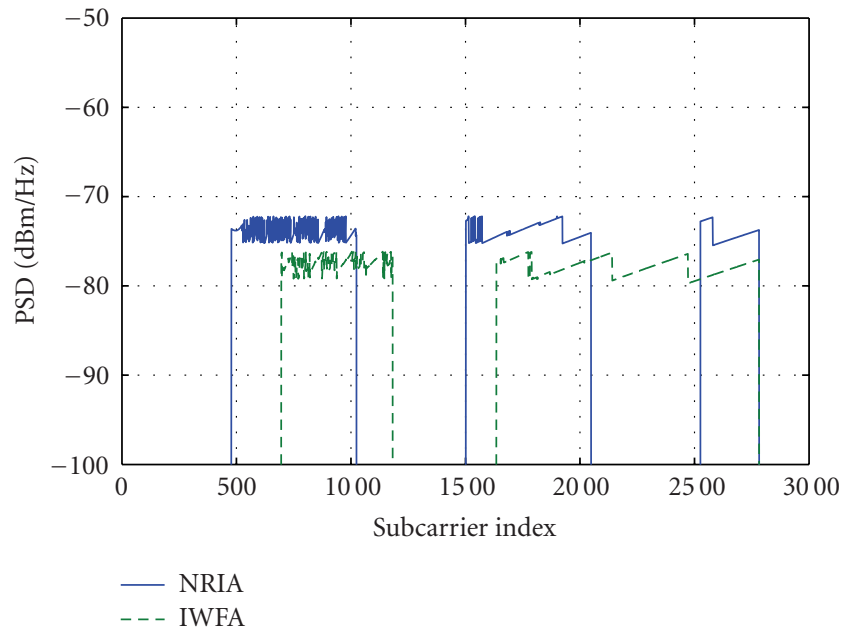

(a)

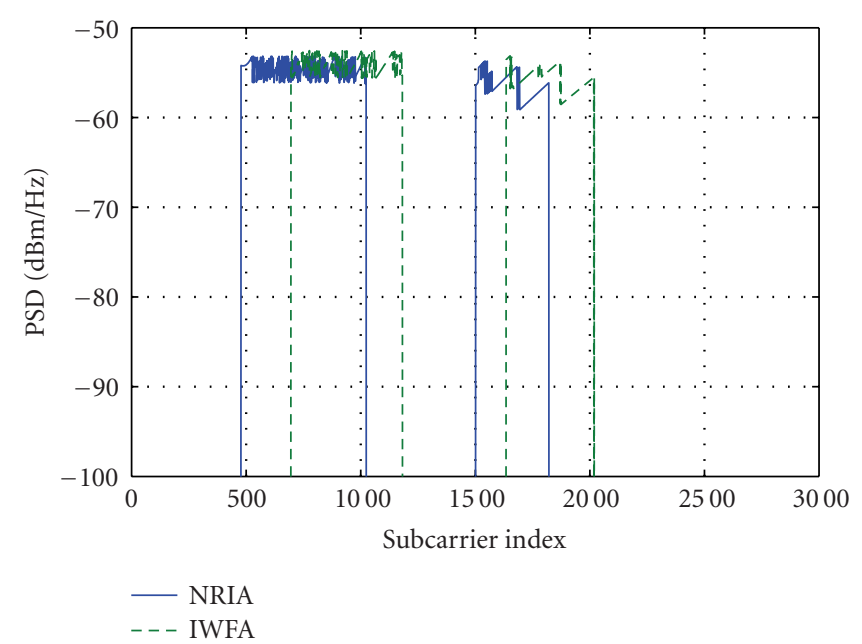

(b)

FIgURe 11: Upstream transmit PSDs of the NRIA and the IWFA for users' bitrates given in Table 3. (a) $u_{1}$ upstream PSDs, (b) $u_{2}$ upstream PSDs.

number of outer iterations is $O_{\max }=10$, when $K=8$ subbands and $N=4096$ subcarriers. The expected number of downstream and upstream inner stage iterations for the twouser case to achieve the desired accuracy is smaller than 50; thus, $i_{\mathrm{DS}}<50$ and $i_{\mathrm{US}}<50$. This statement is confirmed in [3]. Substituting $O=10$ and $i_{\mathrm{DS}}=i_{\mathrm{US}}=50$ into (20) yields

$$
C_{\text {NRIA }}=\mathcal{O}\left(1.5 \cdot 10^{3} \tilde{N}_{\text {DS }}\right)+\mathcal{O}\left(1.5 \cdot 10^{3} \tilde{N}_{\text {US }}\right) .
$$

Correspondingly for the OSBA after substituting $R^{n, \max }=15$ into (22), we get

$$
C_{\mathrm{OSBA}}=\mathcal{O}\left(557.6 \cdot 10^{3} \mathrm{~N}_{\mathrm{DS}}\right)+\mathcal{O}\left(557.6 \cdot 10^{3} \mathrm{~N}_{\mathrm{US}}\right) .
$$

From (23) and (24) we can conclude that also for the two-user case the complexity of the OSBA is much higher than the complexity of the NRIA. When the number of users increases it is obvious from (20) and (22) that the computational complexity of the OSBA increases faster than that of the NRIA. This is because the complexity of the NRIA increases linearly with the number of users, whereas the complexity of the OSBA increases exponentially with the number of users.

Another advantage of the NRIA compared to the OSBA is that the NRIA supports downstream and upstream users' bitrate combinations that the OSBA cannot, due to the optimized downstream and upstream subcarrier allocation. Table 6 summarizes some bitrate combinations that can be supported by the NRIA but not by the OSBA for the selected bandplan "997." Note that these bitrate combinations 


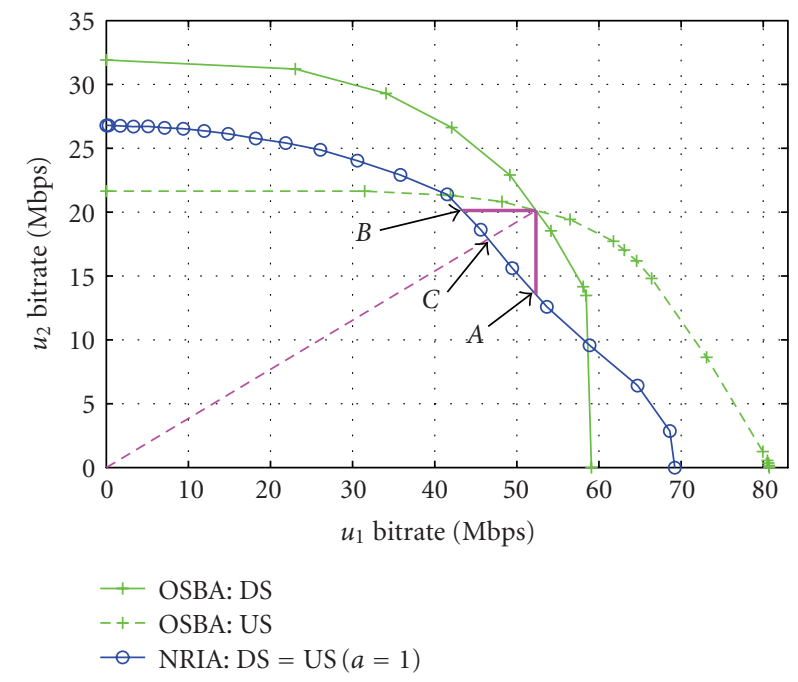

FIGURE 12: Down- and upstream rate regions of the OSBA and NRIA for $a=1, \alpha_{1, \mathrm{DS}}=\alpha_{1, \mathrm{US}}$, and $\alpha_{2, \mathrm{DS}}=\alpha_{2, \mathrm{US}}$.

TABle 4: Comparison of the NRIA with the OSBA, symmetric bitrates $(a=1.00)$.

\begin{tabular}{lcccc}
\hline Algorithm & Direction & $\begin{array}{c}\text { User } u_{1} \\
(\mathrm{Mbps})\end{array}$ & $\begin{array}{c}\text { User } u_{2} \\
(\mathrm{Mbps})\end{array}$ & $\begin{array}{c}\text { Loss } \\
(\%)\end{array}$ \\
\hline OSBA & DS/US & 52.32 & 20.13 & - \\
NRIA $(A)$ & DS/US & 52.32 & 13.55 & 33.3 \\
NRIA $(B)$ & DS/US & 43.37 & 20.13 & 17.2 \\
NRIA $(C)$ & DS/US & 46.52 & 17.90 & 11.1 \\
\hline
\end{tabular}

are generated under the constraint of equal downstream and upstream user priorities.

Figures 14 and 15 show the downstream and upstream transmit PSDs of the OSBA and the NRIA corresponding to asymmetry $a=1.25$ and the bitrates given in Table 5 . The optimized D-FDD bandplan found by the NRIA for this twouser scenario is

$$
\begin{aligned}
I_{\mathrm{DS}} & =\{32 \cdots 493,1025 \cdots 1517,2049 \cdots 2541\}, \\
I_{\mathrm{US}} & =\{494 \cdots 1024,1518 \cdots 2048,2542 \cdots 2782\} .
\end{aligned}
$$

The downstream and upstream PSDs of the NRIA have similar shapes to those shown when comparing the NRIA for $a=1.25$ with the IWFA. However, the downstream and upstream PSDs generated by OSBA have completely different shapes compared to those generated by the IWFA.

Figure 14 shows that the OSBA will partially reduce the transmit power for user $u_{1}$ in the downstream direction. The PSD in the high frequencies of the first downstream subband and in the low frequencies of the second downstream subband is reduced. Therefore, the users are not disturbing each other significantly. In the upstream direction, as can be seen in Figure 15, the PSDs of both users for the OSBA do not overlap at all. They are flat, because the transmitters see more or less flat noise-to-channel-gain ratio $(\mathcal{N} / \mathscr{H}$, cf. Section 2$)$ over all used subcarriers. For a complete comparison,
TABLE 5: Comparison of the NRIA with the OSBA, asymmetric bitrates $(a=1.25)$.

\begin{tabular}{lcccc}
\hline Algorithm & Direction & $\begin{array}{c}\text { User } u_{1} \\
(\mathrm{Mbps})\end{array}$ & $\begin{array}{c}\text { User } u_{2} \\
(\mathrm{Mbps})\end{array}$ & $\begin{array}{c}\text { Loss } \\
(\%)\end{array}$ \\
\hline OSBA & DS & 40.95 & 27.00 & - \\
OSBA & US & 32.76 & 21.60 & - \\
NRIA & DS & 38.95 & 25.68 & 4.9 \\
NRIA & US & 31.29 & 20.63 & 4.5 \\
\hline
\end{tabular}

TABLE 6: Some bitrate combinations that can be supported by the NRIA but not by the OSBA (corresponding to Figures 12 and 13).

\begin{tabular}{lccc}
\hline Asymmetry & Direction & $\begin{array}{c}\text { User } u_{1} \\
(\mathrm{Mbps})\end{array}$ & $\begin{array}{c}\text { User } u_{2} \\
(\mathrm{Mbps})\end{array}$ \\
\hline$a=1.00$ & DS/US & 25.0 & 25.0 \\
$a=1.00$ & DS/US & 35.0 & 23.0 \\
$a=1.00$ & DS/US & 15.0 & 26.0 \\
$a=1.25$ & DS & 25.0 & 28.0 \\
$a=1.25$ & US & 20.0 & 22.5 \\
$a=1.25$ & DS & 13.3 & 29.3 \\
$a=1.25$ & US & 10.2 & 23.3 \\
\hline
\end{tabular}

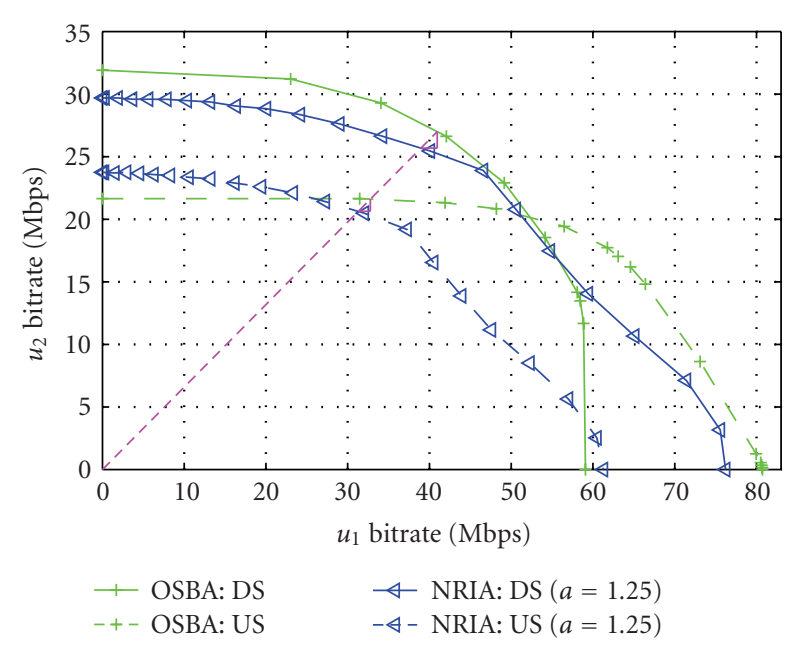

FIGURE 13: Down- and upstream rate regions of the OSBA and NRIA for $a=1.25, \alpha_{1, \mathrm{DS}}=\alpha_{1, \mathrm{US}}$, and $\alpha_{2, \mathrm{DS}}=\alpha_{2, \mathrm{US}}$.

Figure 16 shows the rate regions of all three algorithms (IWFA, OSBA, and NRIA with asymmetry $a=1.25$ ).

\subsection{Comparison of the NRIA with the bidirectional IWFA}

The NRIA, which optimizes the bandplan, always performs better than IWFA, which assumes a fixed bandplan. As an alternative to the IWFA, Cioff [8] has suggested to compare the NRIA with the bidirectional IWFA (bi-IWFA). The bi-IWFA does not fix the bandplan, but assumes an echocancelled transmission scheme and lets the IWFA decide for each loop which subcarriers should be used exclusively for 


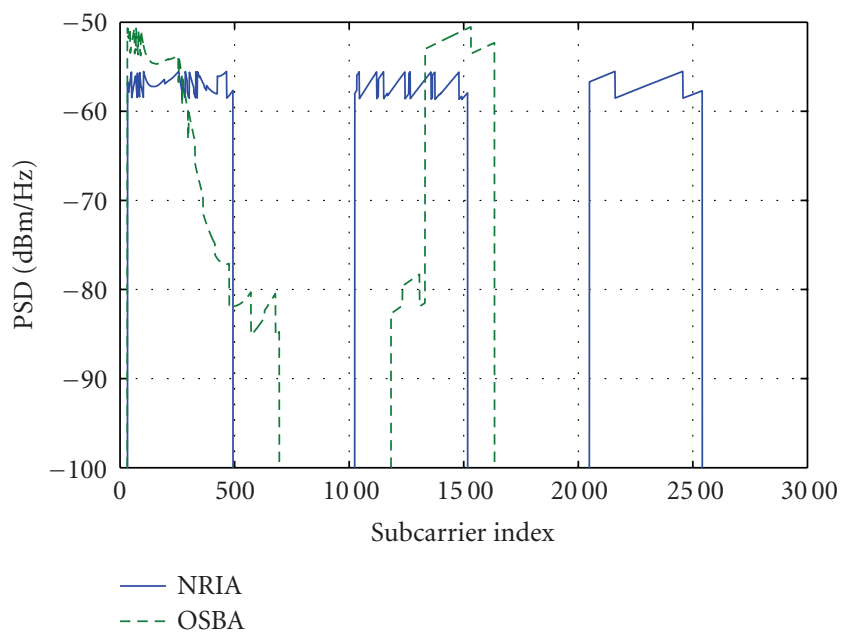

(a)

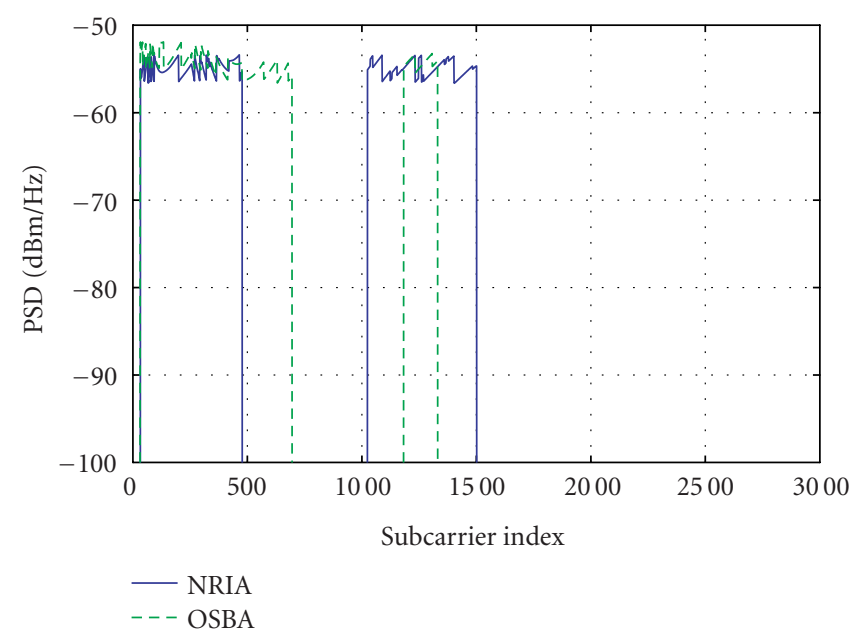

(b)

FIgURe 14: Downstream transmit PSDs of the NRIA and the OSBA for users' bitrates given in Table 5. (a) $u_{1}$ downstream PSDs; (b) $u_{2}$ downstream PSDs.

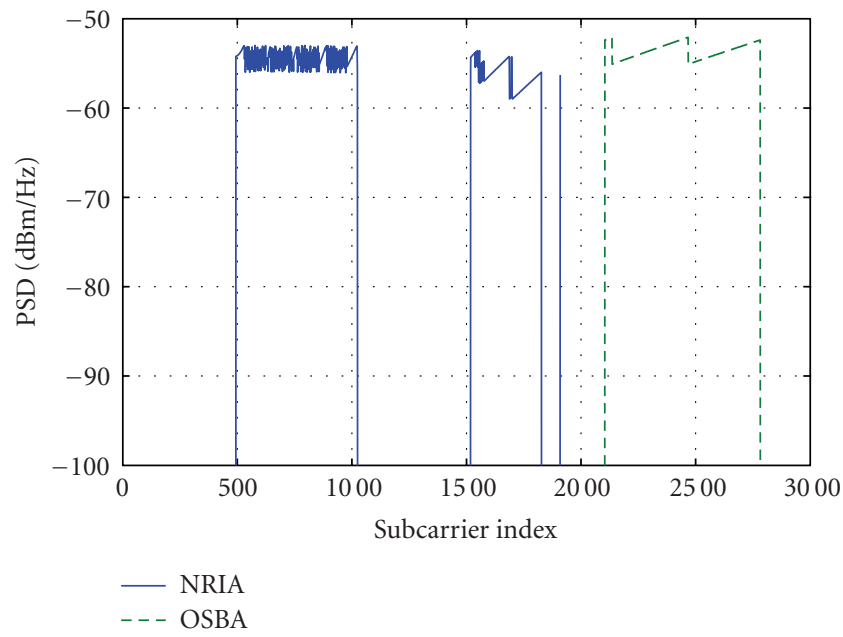

(a)

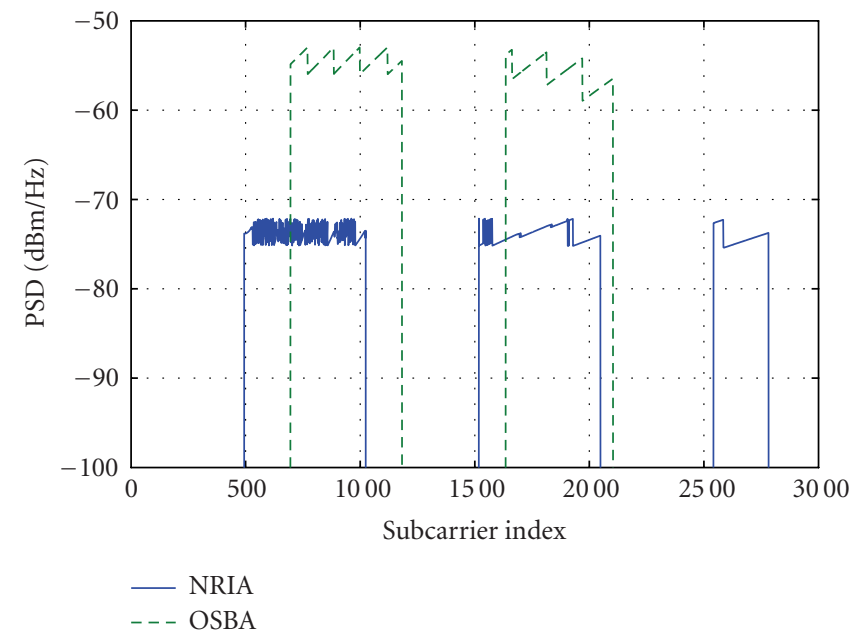

(b)

FIGURE 15: Upstream transmit PSDs of the NRIA and the OSBA for users' bitrates given in Table 5. (a) $u_{1}$ upstream PSDs; (b) $u_{2}$ upstream PSDs.

downstream or upstream and which should be used simultaneously for both transmission directions.

The simulation scenario used to compare the NRIA with the bi-IWFA is shown in Figure 17. We have selected a network scenario with four users, because in a two-user case an echo-cancellation (EC) scheme will usually outperform any another algorithm that assumes FDD transmission. The reason for this is that the gain achieved with EC is higher than the loss from the NEXT noise of a single disturber.

When the number of users $U>2$, [22] has proved that the IWFA converges and has a unique Nash equilibrium if

$$
\max \left\{\Gamma \frac{\mathscr{H}_{u v}^{n}}{\mathscr{H}_{u u}^{n}}\right\}<\frac{1}{U-1}, \quad \forall n, u, v \text { and } u \neq v,
$$

where $\Gamma, \mathscr{H}_{u v}^{n}$, and $\mathscr{H}_{u u}^{n}$ are the same as defined in Section 2. Note, however, that here $\mathscr{H}_{u v}^{n}$ can be NEXT and FEXT couplings. For the network scenario in Figure 17 the criterion (26) is not always fulfilled due to high NEXT couplings. Hence, the bi-IWFA might not have a unique Nash equilibrium $[4,22]$. For this scenario, we have observed that the performance of the bi-IWFA depends on the user ordering during the iterations. Therefore, we have performed simulations with two iteration orderings: $u_{1}-u_{2}-u_{3}-u_{4}$ and $u_{4}-u_{3}-u_{2}-u_{1}$.

For these simulations we have searched for symmetric and equal bitrates for all users. The simulation results are summarized in Figure 18. When the bi-IWFA is deployed bitrates of approximately 26.2 Mbps and 24.6 Mbps can be supported by each user for the iteration orders $u_{4}-u_{3}-u_{2}-u_{1}$ and 


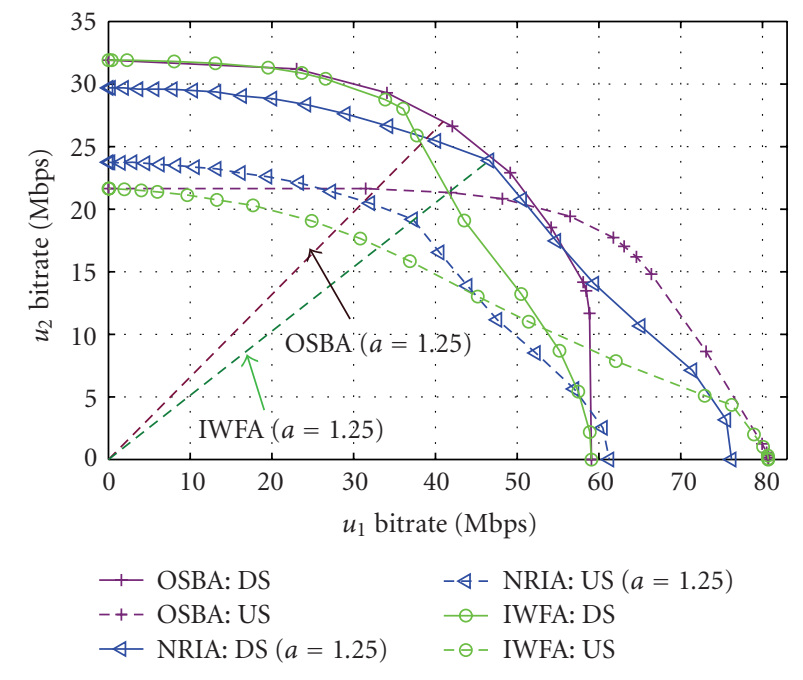

FIGURE 16: Down- and upstream rate regions of the IWFA, OSBA, and NRIA for $a=1.25, \alpha_{1, \mathrm{DS}}=\alpha_{1, \mathrm{US}}$, and $\alpha_{2, \mathrm{DS}}=\alpha_{2, \mathrm{US}}$.

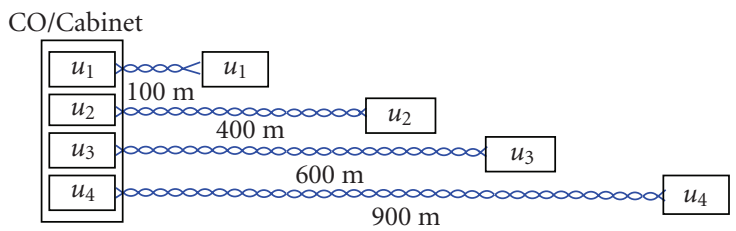

FIgURE 17: Network scenario used to compare the NRIA and the bi-IWFA.

$u_{1}-u_{2}-u_{3}-u_{4}$, respectively. With the NRIA a bitrate of more than $31.6 \mathrm{Mbps}$ can be achieved by all users in each transmission direction. Thus, for this case a bitrate increase of more than $20 \%$ is achieved with the NRIA for each user compared to the bi-IWFA with the best iteration order $u_{4}-u_{3}$ $u_{2}-u_{1}$. This simulation is also performed with the IWFA using the bandplan "997," as described in Section 6.2. Figure 18 shows that the IWFA performs better than the bi-IWFA for the given scenario. The reason for this can be explained as follows: due to the several NEXT couplings the crosstalk noise is high; however, the noise level at the receivers is not so high that the algorithm decides for FDD transmission when the transmit PSDs of all transmitters are "moderately" low. As Chung [7] recognized, in these environments the IWFA shows significantly worse performance compared to the case where the PSDs have high levels.

The downstream and upstream transmit PSDs of the bi-IWFA for the iteration order $u_{4}-u_{3}-u_{2}-u_{1}$ are shown in Figure 19. The transmit PSDs for the iteration order $u_{1}-u_{2}$ $u_{3}-u_{4}$ are not included, but were found to have quite different shapes. Figure 19 shows that only user $u_{4}$ utilizes the maximum total power for the upstream direction. Therefore, $u_{4}$ determines the maximum bitrates of all other users. From the PSDs we can recognize three main regions: frequencies lower than approximately $6.2 \mathrm{MHz}$ (subcarrier 1438) are used

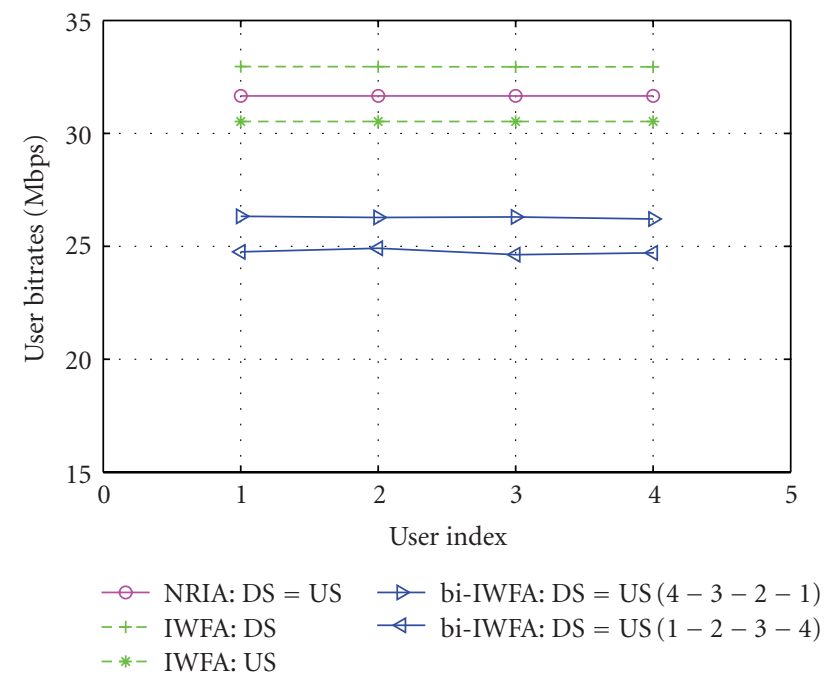

FIGURE 18: Users' downstream and upstream supported bitrates for NRIA and bi-IWFA for the network scenario shown in Figure 17.

simultaneously for both transmission directions; frequencies between $6.2 \mathrm{MHz}$ and 10.4 MHz (subcarrier 2405) are used only for downstream transmission; and frequencies from 10.4 MHz to $12 \mathrm{MHz}$ (subcarrier 2782) are used simultaneously for both transmission directions. Figure 20 shows that in contrast to the bi-IWFA, the NRIA allows user $u_{4}$ to utilize the maximum total power in both transmission directions.

\section{SUMMARY}

Dynamic spectrum management (DSM) should use as many degrees of freedom as possible in order to optimize the utilization of cable resources for DSL more efficiently. Previously proposed DSM algorithms for frequency division duplexing (FDD) systems consider only user power allocation for a fixed downstream and upstream frequency bandplan, albeit a dynamic spectrum often helps to utilize the cable capacity more efficiently.

In this paper, we presented a novel centralized DSM algorithm for DSL: the normalized-rate iterative algorithm (NRIA). The NRIA is the only algorithm that jointly optimizes the bandplan for FDD systems and power allocations for all users in a common cable bundle.

The multiuser optimization performed by the NRIA is based on a unique problem formulation that has a strong practical advantage. It is based on two types of parameters, which bridge the gap between the operators' DSL business models and the DSM: the desired user priorities and the desired network asymmetry. The NRIA offers high performance in combination with low computational complexity, since it is designed to be practically implementable rather than just obtaining the highest theoretical performance.

An inner iteration stage of the NRIA is based on the seemingly practical iterative water-filling algorithm (IWFA) [4] for finding efficient users' power allocations. However, the NRIA extends on the IWFA by automatically finding the 


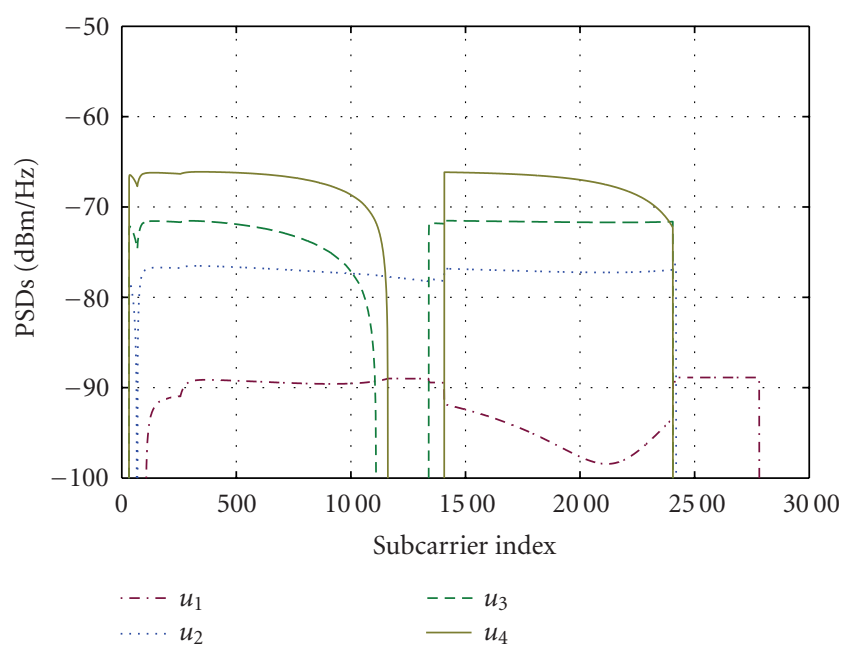

(a)

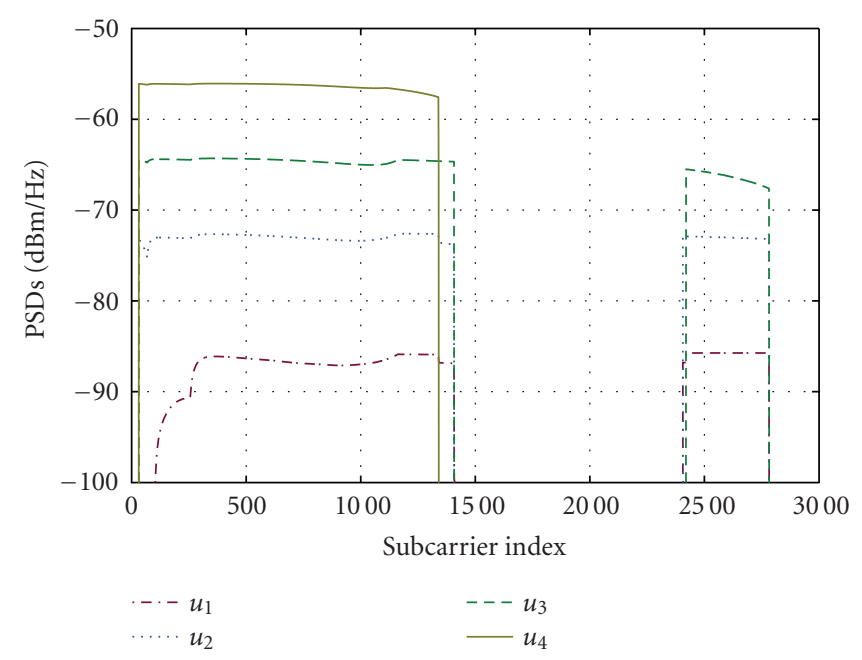

(b)

FIGURE 19: (a) The downstream and (b) upstream transmit PSDs of the bi-IWFA for users' bitrates shown in Figure 18 when the iteration order $u_{4}-u_{3}-u_{2}-u_{1}$ has been selected.

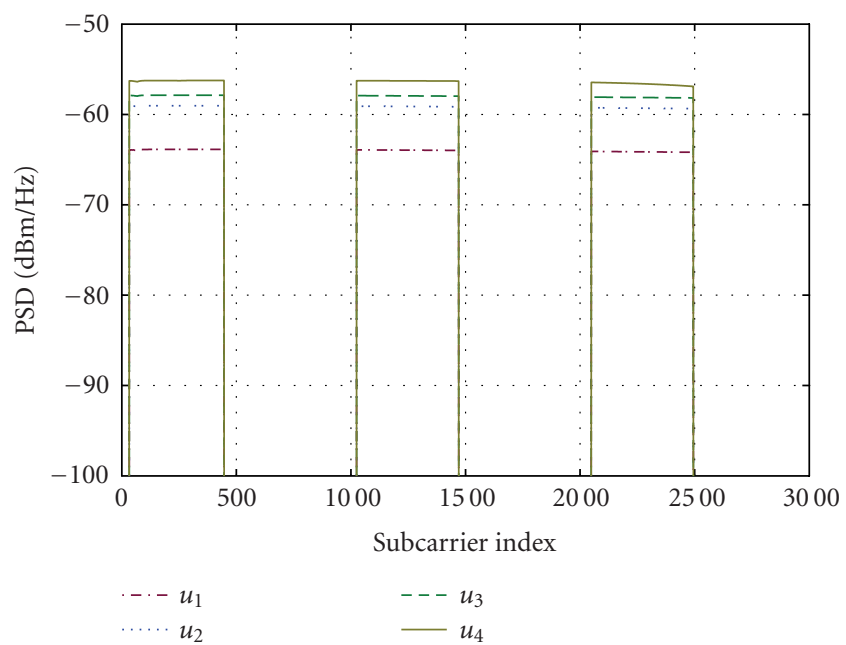

(a)

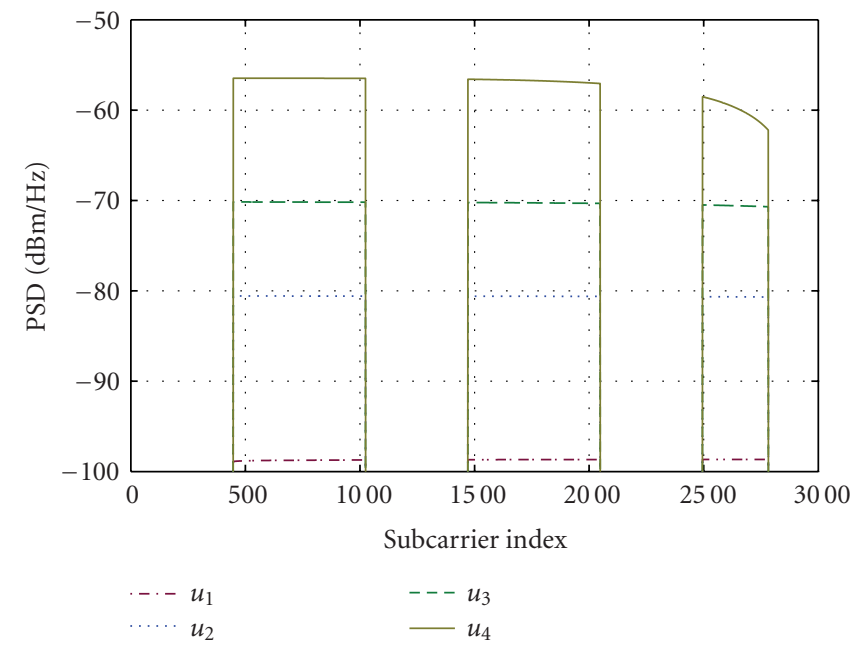

(b)

FIgURe 20: (a) The downstream and (b) upstream transmit PSDs of the NRIA for users' bitrate values shown in Figure 18.

users' bitrates that are actually achievable. This is accomplished by taking advantage of the property that the NRIA is centralized which enables user cooperation. Furthermore, an outer iteration stage uses a simple but effective search strategy for finding an effective bandplan. All these practical advantages combined make the NRIA attractive also for networks comprised of many DSL users.

Simulations showed that the NRIA achieves better bitrate performance than both the IWFA and the bidirectional IWFA [8]. The NRIA can achieve almost as good performance as the optimal spectrum balancing algorithm [5], but with much lower requirements on complexity. However, by utilizing the additional feature of an optimized bandplan, the
NRIA can offer bitrate combinations, that is, DSL services, that cannot be offered by any other DSM algorithm.

\section{APPENDIX}

\section{A. VALIDITY OF POSTULATE 1}

In this appendix we will discuss the validity of Postulate 1 given in Section 5.1, "check the convergence point" stating: "Consider a multiuser D-FDD transmission system operating in an interference channel where each receiver considers the crosstalk signal as noise. For such a multiuser system the sum of the user bitrates increases when the power of each user increases." 
Using Theorem A.1 below, we show that Postulate 1 is true for the case when all subcarriers are utilized by all users under the assumption that the receivers operate with high signal-to-noise ratio (SNR). In our experience, Postulate 1 is also true when not all subcarriers are used. Furthermore, in [23] it is shown that for a theoretical two-user Gaussian interference channel and different coupling values the sum of the bitrates is increased by increasing the power of the two users.

Theorem A.1. Assume that receivers operate with high SNR. If all subcarriers are utilized by all users, the users consider the crosstalk signal as a Gaussian noise and none of the users utilize the maximum total power, the sum of the user bitrates always increases when the power of each user increases. That is,

$$
\sum_{u} \hat{R}_{u}>\sum_{u} R_{u}
$$

where $\hat{R}_{u}$ and $R_{u}$ denote the bitrates of user $u$ with and without power increase, respectively.

Proof. Without loss of generality we prove Theorem A.1 for two users. The proof for the case with more than two users is a generalization of this case. First, (A.1) can be written as

$$
\widehat{R}_{1}+\widehat{R}_{2}>R_{1}+R_{2} \text {. }
$$

The power allocations that correspond to $R_{1}$ and $R_{2}$ are: $\mathcal{P}_{1}=\left[\mathcal{P}_{1}^{0}, \mathcal{P}_{1}^{1}, \ldots, \mathcal{P}_{1}^{N-1}\right]$ and $\mathcal{P}_{2}=\left[\mathcal{P}_{2}^{0}, \mathcal{P}_{2}^{1}, \ldots, \mathcal{P}_{2}^{N-1}\right]$, respectively. In the same way we will denote the increased power levels that correspond to $\widehat{R}_{1}$ and $\widehat{R}_{2}$ with $\widehat{\mathcal{P}}_{1}=$ $\left[\widehat{\mathcal{P}}_{1}^{0}, \widehat{\mathcal{P}}_{1}^{1}, \ldots, \widehat{\mathcal{P}}_{1}^{N-1}\right]$ and $\widehat{\mathcal{P}}_{2}=\left[\widehat{\mathcal{P}}_{2}^{0}, \widehat{\mathcal{P}}_{2}^{1}, \ldots, \widehat{\mathcal{P}}_{2}^{N-1}\right]$. We will now proceed to show that Theorem A.1 follows as a consequence of the increase in $\widehat{\mathcal{P}}_{1}$ and $\widehat{\mathcal{P}}_{2}$.

First we note that increasing the bits in each subcarrier individually also increases their sum. That is, it is enough to study a particular subcarrier $n$ in (A.2):

$$
\widehat{R}_{1}^{n}+\widehat{R}_{2}^{n}>R_{1}^{n}+R_{2}^{n},
$$

where all the bitrates are calculated based on (4).

The SNR at the receivers is much greater than one over all subcarriers due to our assumption that receivers operate with high SNR (this assumption is always true for the subcarriers that are utilized for data transmission). Under this assumption we can expand (A.3) using (4) and (5) to

$$
\begin{aligned}
\log _{2}\left(\frac{\mathcal{H}_{11}^{n} \widehat{\mathcal{P}}_{1}^{n}}{\Gamma\left(\mathscr{H}_{12}^{n} \widehat{\mathcal{P}}_{2}^{n}+\mathcal{P}_{V}^{n}\right)}\right)+\log _{2}\left(\frac{\mathcal{H}_{22}^{n} \widehat{\mathcal{P}}_{2}^{n}}{\Gamma\left(\mathscr{H}_{21}^{n} \widehat{\mathcal{P}}_{1}^{n}+\mathcal{P}_{V}^{n}\right)}\right) \\
\quad>\log _{2}\left(\frac{\mathscr{H}_{11}^{n} \mathcal{P}_{1}^{n}}{\Gamma\left(\mathscr{H}_{12}^{n} \mathcal{P}_{2}^{n}+\mathcal{P}_{V}^{n}\right)}\right)+\log _{2}\left(\frac{\mathcal{H}_{22}^{n} \mathcal{P}_{2}^{n}}{\Gamma\left(\mathscr{H}_{21}^{n} \mathcal{P}_{1}^{n}+\mathcal{P}_{V}^{n}\right)}\right) .
\end{aligned}
$$

Using the properties of the logarithm we can rewrite this as

$$
\begin{aligned}
& \frac{\widehat{\mathcal{P}}_{1}^{n} \widehat{\mathcal{P}}_{2}^{n}}{\left(\mathscr{H}_{12}^{n} \widehat{\mathcal{P}}_{2}^{n}+\mathcal{P}_{V}^{n}\right)\left(\mathscr{H}_{21}^{n} \widehat{\mathcal{P}}_{1}^{n}+\mathcal{P}_{V}^{n}\right)} \\
& >\frac{\mathcal{P}_{1}^{n} \mathcal{P}_{2}^{n}}{\left(\mathscr{H}_{12}^{n} \mathcal{P}_{2}^{n}+\mathcal{P}_{V}^{n}\right)\left(\mathscr{H}_{21}^{n} \mathcal{P}_{1}^{n}+\mathcal{P}_{V}^{n}\right)},
\end{aligned}
$$

or equivalently,

$$
\frac{\widehat{\mathcal{P}}_{1}^{n}}{\mathcal{P}_{1}^{n}} \cdot \frac{\widehat{\mathcal{P}}_{2}^{n}}{\mathcal{P}_{2}^{n}}>\frac{\mathscr{H}_{21}^{n} \widehat{\mathcal{P}}_{1}^{n}+\mathcal{P}_{V}^{n}}{\mathscr{H}_{21}^{n} \mathcal{P}_{1}^{n}+\mathcal{P}_{V}^{n}} \cdot \frac{\mathscr{H}_{12}^{n} \widehat{\mathcal{P}}_{2}^{n}+\mathcal{P}_{V}^{n}}{\mathscr{H}_{12}^{n} \mathcal{P}_{2}^{n}+\mathcal{P}_{V}^{n}}
$$

In (A.6) we can identify the part that relates to the first user as

$$
\frac{\widehat{\mathcal{P}}_{1}^{n}}{\mathcal{P}_{1}^{n}}>\frac{\mathscr{H}_{21}^{n} \widehat{\mathcal{P}}_{1}^{n}+\mathcal{P}_{V}^{n}}{\mathscr{H}_{21}^{n} \mathcal{P}_{1}^{n}+\mathcal{P}_{V}^{n}}
$$

From (A.7) we can derive

$$
\widehat{\mathcal{P}}_{1}^{n}>\mathcal{P}_{1}^{n}
$$

A corresponding relation is found for the second user and, since this is true for both users, (A.6) is always true. Thus, the left-hand side of (A.2) is always larger than the right-hand side.

Note that when $\mathscr{H}_{21}^{n} \widehat{\mathcal{P}}_{1}^{n} \gg \mathcal{P}_{V}^{n}$ and $\mathscr{H}_{21}^{n} \mathcal{P}_{1}^{n} \gg \mathcal{P}_{V}^{n}$ the left-hand side of (A.7) is only slightly larger than the righthand side. This means that there is only a minor increase in the sum of users' bitrates when the power of the signal is increased. Furthermore, when $\mathcal{P}_{V}^{n}=0$, both sides in (A.7) are equal. Thus, there is no increase in the sum of the user bitrates when the power of each user increases. However, this is not important for communication over copper wires, because $\mathcal{P}_{V}^{n}$ is never zero due to the thermal noise on copper, external noise sources such as radio noise, and also alien noise from the other DSL systems not included in the optimization process.

\section{ACKNOWLEDGMENT}

This work was partially financed by the Austrian Kplus program.

\section{REFERENCES}

[1] D. Statovci and T. Nordström, "Adaptive subcarrier allocation, power control, and power allocation for multiuser FDD-DMT systems," in Proceedings of IEEE International Conference on Communications (ICC '04), vol. 1, pp. 11-15, Paris, France, June 2004.

[2] D. Statovci and T. Nordström, "Adaptive resource allocation in multiuser FDD-DMT systems," in Proceedings of 12th European Signal Processing Conference (EUSIPCO '04), pp. 12131216, Vienna, Austria, September 2004.

[3] D. Statovci, Adaptive resource allocation for multi-user digital subscriber lines, Ph.D. thesis, Vienna University of Technology, Vienna, Austria, July 2005.

[4] W. Yu, G. Ginis, and J. M. Cioffi, "Distributed multiuser power control for digital subscriber lines," IEEE Journal on Selected Areas in Communications, vol. 20, no. 5, pp. 1105-1115, 2002.

[5] R. Cendrillon, M. Moonen, J. Verliden, T. Bostoen, and W. $\mathrm{Yu}$, "Optimal multiuser spectrum management for digital subscriber lines," in Proceedings of IEEE International Conference on Communications (ICC '04), vol. 1, pp. 1-5, Paris, France, June 2004. 
[6] R. Cendrillon, Multi-user signal and spectra co-ordination for digital subscriber lines, Ph.D. thesis, Department of Electrical Engineering, Katholieke Universiteit Leuven, Leuven, Belgium, December 2004.

[7] S. T. Chung and J. M. Cioffi, "Rate and power control in a two-user multicarrier channel with no coordination: the optimal scheme versus a suboptimal method," IEEE Transactions on Communications, vol. 51, no. 11, pp. 1768-1772, 2003.

[8] J. M. Cioffi, "Use of bi-directional iterative water-filling," Personal communication, Vienna, Austria, September 2004.

[9] F. Sjöberg, M. Isaksson, R. Nilsson, P. Ödling, S. K. Wilson, and P. O. Börjesson, "Zipper: a duplex method for VDSL based on DMT," IEEE Transactions on Communications, vol. 47, no. 8, pp. 1245-1252, 1999.

[10] R. Nilsson, F. Sjöberg, M. Isaksson, J. M. Cioffi, and S. K. Wilson, "Autonomous synchronization of a DMT-VDSL system in unbundled networks," IEEE Journal on Selected Areas in Communications, vol. 20, no. 5, pp. 1055-1063, 2002.

[11] F. Sjöberg, R. Nilsson, M. Isaksson, P. Ödling, and P. O. Börjesson, "Asynchronous Zipper," in Proceedings of IEEE International Conference on Communications (ICC '99), vol. 1, pp. 231-235, Vancouver, BC, Canada, June 1999.

[12] C. A. Floudas, Nonlinear and Mixed-Integer Optimization: Fundamentals and Applications, Oxford University Press, New York, NY, USA, 1995.

[13] I. E. Grossmann, "Review of nonlinear mixed-integer and disjunctive programming techniques," Optimization and Engineering, vol. 3, no. 3, pp. 227-252, 2002.

[14] ETSI, "Transmission and Multiplexing (TM); Access transmission systems on metallic access cables; Very high speed Digital Subscriber Line (VDSL); Part 1: Functional requirements," ETSI Standard TS 101 270-1, Version 1.3.1, July 2003.

[15] ETSI, "Transmission and Multiplexing (TM); Access transmission systems on metallic access cables; Very high speed Digital Subscriber Line (VDSL); Part 2: Transceiver specification," ETSI Standard TS 101 270-2, Version 1.2.1, July 2003.

[16] ANSI, "Very-high bit-rate Digital Subscriber Lines (VDSL) Metallic Interface, Part 3: Technical Specification of a Multi-Carrier Modulation Transceiver," ANSI Draft Standard T1.424/Trial-Use Part3, November 2000.

[17] T. Starr, M. Sorbara, J. M. Cioffi, and P. Silverman, DSL Advances, Prentice-Hall, Upper Saddle River, NJ, USA, 2003.

[18] J. Campello, "Practical bit loading for DMT," in Proceedings of IEEE International Conference on Communications (ICC '99), vol. 2, pp. 801-805, Vancouver, BC, Canada, June 1999.

[19] R. F. M. van den Brink, "Cable reference models for simulating metallic access networks," ETSI/STC TM6 contribution 970p02r3, June 1998.

[20] J. Verlinden, "The target PSD obtained with iterative waterfilling is almost flat," ANSI T1E1.4 contribution 2003-295, December 2003.

[21] R. Suciu, E. Van den Bogaert, J. Verlinden, and T. Bostoen, "Insuring spectral compatibility of iterative water-filling," in Proceedings of 12th European Signal Processing Conference (EUSIPCO '04), vol. 1, pp. 1209-1212, Vienna, Austria, September 2004.

[22] S. T. Chung, S. J. Kim, J. Lee, and J. M. Cioffi, "A gametheoretic approach to power allocation in frequency-selective Gaussian interference channels," in Proceedings of IEEE International Symposium on Information Theory (ISIT '03), pp. 316-316, Yokohama, Japan, June-July 2003.

[23] M. H. M. Costa, "On the Gaussian interference channel," IEEE Transactions on Information Theory, vol. 31, no. 5, pp. 607615, 1985.
Driton Statovci was born in Batllavë, Kosova, in 1972. He received the "Inxh. Dipl." degree (equivalent to a master's degree) in 1996 from the University of Prishtina, Faculty of Electrical Engineering, Stream of Informatics and Telecommunications. He received the Ph.D. degree in 2005 from Vienna University of Technology, Austria. From August 2000 until January 2002, he joined Ahead Communications Systems. At

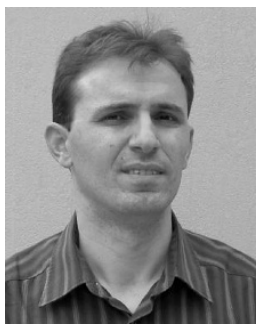
the same time, he was delegated by Ahead Communications Systems as a Researcher at the Telecommunications Research Center Vienna (ftw.), working on the Broadband Access over Wire project. At Ahead Communications Systems he worked in system design for transmitting data and voice over DSL access networks. In February 2002, he joined the Telecommunications Research Center Vienna (ftw.), Austria. Currently he is working as Researcher in the "Active Copper Resource Management, ARM-Cu" project. His current research interests include multiuser transmission theory and optimization of resource utilization in wireline and wireless communications.

Tomas Nordström was born in Härnösand, Sweden, in 1963. He received the M.S.E.E. degree in 1988, the Licentiate degree in 1991, and the Ph.D. degree in 1995, all from Luleå University of Technology, Sweden. Currently, he is a Key Researcher and Project Manager at the Telecommunications Research Center Vienna (ftw.). During 1995 and 1996, he was an Assistant Professor at Luleå University of Technology re-

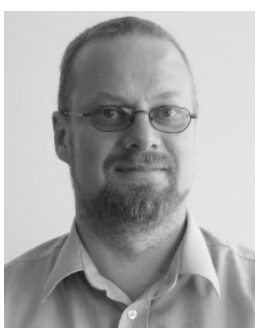
searching computer architectures, neural networks, and signal processing. Between 1996 and 1999, he was with Telia Research AB (the research branch of the Swedish incumbent telephone operator) where he developed broadband Internet communication over twisted copper pairs. He was instrumental in the development of the Zipper-VDSL concept (contributed to the standardization of VDSL in ETSI, ANSI, and ITU) and in the design of the ZipperVDSL prototype modems. In addition, he was Telia's National Expert on speaker verification. In December 1999, he joined the Telecommunications Research Center Vienna ( $\mathrm{ftw}$.), where he is the Project Manager of the "Broadband Wireline Access" group. At FTW, he has worked with various aspects of wireline communications like simulation of xDSL systems, cable measurements, RFI suppression, exploiting the common-mode signal in xDSL, and dynamic spectrum management. He is also a Consultant covering design, development, and deployment of DSL modems.

Rickard Nilsson was born in Umeå, Sweden, in 1971. He received the M.S.E.E. degree, in 1996, the Licentiate of engineering, in 1999, and the Ph.D. degree in signal processing, in January 2002, all from Luleå University of Technology, Luleå, Sweden. Between 1997 and 2001, he worked cooperatively with Telia Research AB, Sweden, by contributing to the development and standardization of the DMT-Zipper method for

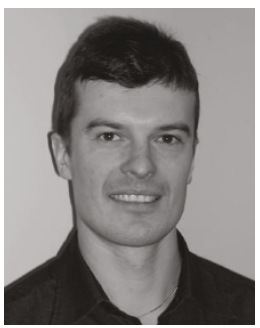
VDSL. During the fall semester in 1999, he was a Guest Researcher at Stanford University, USA. In May 2002, he moved from northern Sweden to Austria and joined the Telecommunications Research Center Vienna (ftw.). His research interests include multicarrier modulation, statistical signal processing, low-complexity algorithms, and interference suppression and synchronization. 